\title{
From Pythagoras Theorem to Fermat's Last Theorem and the Relationship between the Equation of Degree $n$ with One Unknown
}

\author{
Yufeng Xia \\ Huaqiao University, Fujian, China \\ Email: qhdxxyf1991@163.com
}

How to cite this paper: Xia, Y.F. (2020) From Pythagoras Theorem to Fermat's Last Theorem and the Relationship between the Equation of Degree $n$ with One Unknown. Advances in Pure Mathematics, 10, 125-154. https://doi.org/10.4236/apm.2020.103009

Received: August 27, 2019

Accepted: March 22, 2020

Published: March 25, 2020

Copyright ( 2020 by author(s) and Scientific Research Publishing Inc. This work is licensed under the Creative Commons Attribution International License (CC BY 4.0).

http://creativecommons.org/licenses/by/4.0/

(c) (i) Open Access

\begin{abstract}
The most interesting and famous problem that puzzled the mathematicians all around the world is much likely to be the Fermat's Last Theorem. However, since the Theorem was proposed, people can't find a way to solve the problem until Andrew Wiles proved the Fermat's Last Theorem through a very difficult method called Modular elliptic curves in 1995. In this paper, I firstly constructed a geometric method to prove Fermat's Last Theorem, and in this way we can easily get the conclusion below: If $a$ and $b$ are integer and $a=b, n \in \mathbb{Q}$ and $n>1$, the value of $c$ satisfies the function $a^{n}+b^{n}=c^{n}$ that can never be integer; if $a, b$ and $c$ are integer and $a \neq b$, $n$ is integer and $n>2$, the function $a^{n}+b^{n}=c^{n}$ cannot be established.
\end{abstract}

\section{Keywords}

Pythagoras Theorem, Fermat's Last Theorem, Geometric Method, Equation of Degree $n$ with One Unknown

\section{Introduction}

The Fermat's Last Theorem was proposed by French famous mathematician Pierre de Fermat in 1637, it was called the last theorem because it was the theorem of Fermat that can be proved at last, which means to prove the theorem is very difficult. The Fermat's Last Theorem states: there is no positive integer $a, b$ and $c$ to satisfy the function $a^{n}+b^{n}=c^{n} \quad$ (1) when $n$ is integer and $n>2$ [1].

Many mathematicians paid attention to this theorem, and they found it not as easy as it looks like. In 1753, the famous Swiss mathematician Euler said in a letter to Goldbach that he proved the Fermat conjecture at $n=3$, and his proof was published in the book Algebra Guide in 1770 [2]. Fermat himself proved the Fermat conjecture at $n=4$ [3]. In 1825, the German mathematician Dirichlet 
and the French mathematician Legendre independently proved that Fermat's theorem was established at $n=5$, using the extension of the method used by Eulerasin [2]. In 1844, Kummer proposed the concept of "ideal number", he proved that for all prime indices $n$ less than 100, Fermat's theorem was established, and this study came to a stage [4]. But the mathematicians still struggled with Fermat's theorem in the first two hundred years of the conjecture with little progress. What's more, many theorem were proposed in order to prove the Fermat's Last Theorem, such as Modell conjecture, Taniyama-Shimura theorem. After proving the Taniyama-Shimura theorem, Andrew Wiles finally got a way to prove the Fermat's Last Theorem in 1995 [5].

At first, people wanted to prove that the Fermat's Last Theorem was established at different indices $n$, but the indices $n$ is infinite, this method is meant to be failed. Then, people tried to propose another theorem to indirectly prove the Fermat's Last Theorem, but the relationship between two theorems is not very clear, thus the proof is hard to be verified.

To prove the Fermat's Last Theorem, I got inspiration from the Pythagoras Theorem. As we all know the Pythagoras Theorem: the sum of the squares of the two right-angled sides of a right-angled triangle is equal to the square of the hypotenuse, let the length of two right-angled sides be $a$ and $b$, and the length of hypotenuse is $c$, then $a^{2}+b^{2}=c^{2}$ (2) [6]. What's more, if $a, b$ and $c$ satisfy the function (2), the angle $(\theta)$ between $a$ and $b$ must be $\frac{\pi}{2}$. If $a, b$ and $c$ satisfy the function $a^{n}+b^{n}=c^{n} \quad(1)$, what is the relationship between $n$ and $\theta$ ? This paper discusses the relationship between $n$ and $\theta$, and in this geometric method, we can easily prove the Fermat's Last Theorem.

\section{Proof}

\subsection{Geometric Construction}

A triangle has three sides, $a, b$ and $c$, respectively. Firstly, let us discuss an easy condition: $a=b$.

As we can see in Figure 1, the point $\mathrm{O}$ is the center of circle, the radius of the circle is $r$, the point $\mathrm{A}$ and $\mathrm{B}$ are on the circle, and A is fixed, B can move to $\mathrm{B}^{\prime}$ ( $\mathrm{B}^{\prime}$ is $\mathrm{B} 1, \mathrm{~B} 2, \ldots$, shown on the circle), connect point $\mathrm{O}, \mathrm{A}$ and $\mathrm{B}$ to form a triangle $\triangle \mathrm{OAB}$. The length of each side of $\triangle \mathrm{OAB}$ is: $\mathrm{OA}=a, \mathrm{OB}=b, \mathrm{AB}=c$, and $a=b=r$, the angle of $\angle \mathrm{BOB}^{\prime}=\theta$.

If $\angle \mathrm{BOB}^{\prime}=\theta=0 \quad\left(\mathrm{~B}^{\prime}=\mathrm{B}\right)$, then $\quad \mathrm{AB}=c=a+b=2 r, a, b$ and $c$ satisfy the function $a^{n}+b^{n}=c^{n} \quad$ (2), in this condition, $n=1$.

If $\angle \mathrm{BOB}^{\prime}=\theta=\frac{\pi}{2} \quad\left(\mathrm{~B}^{\prime}=\mathrm{B} 3\right)$, according to Pythagoras Theorem:

$\mathrm{AB}^{2}=c^{2}=a^{2}+b^{2}, \mathrm{a}, \mathrm{b}$ and $\mathrm{c}$ satisfy the function $a^{n}+b^{n}=c^{n} \quad(1)$, in this condition, $n=2$.

In general condition: $\angle \mathrm{BOB}^{\prime}=\theta$, because of $\mathrm{BB}^{\prime} \perp \mathrm{AB}^{\prime}$, then $c=2 r \cos \frac{\theta}{2}$, if $a, b$ and $c$ satisfy the function $a^{n}+b^{n}=c^{n} \quad(1)$, then 


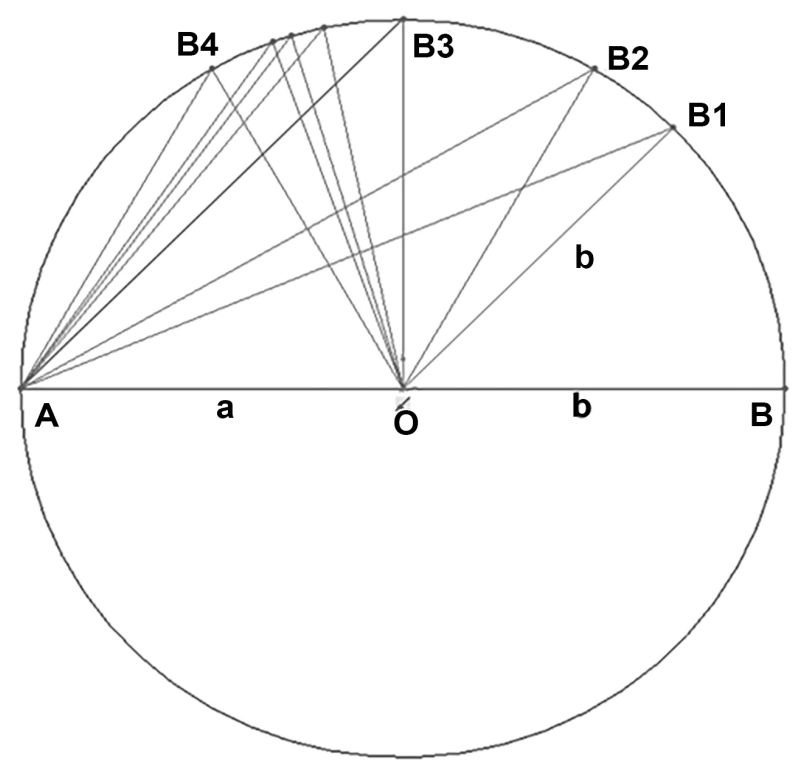

Figure 1. The Geometric construction to prove Fermat's Last Theorem $(a=b)$.

$$
\begin{gathered}
2 r^{n}=\left(2 r \cos \frac{\theta}{2}\right)^{n} \\
2^{\frac{1}{n}}=2 \cos \frac{\theta}{2} \\
\frac{1}{n}=\log _{2}\left(2 \cos \frac{\theta}{2}\right)=\log _{2}\left(\frac{c}{r}\right)
\end{gathered}
$$

The function (3) shows the relationship between $n$ and $\theta$, function (3) is the necessary and sufficient conditions of function (1). We can draw the function (3) as Figure 2. $(\theta \in[0, \pi])$.

1) When $\theta=\frac{2 \pi}{3}-\epsilon,(\epsilon>0$ and $\epsilon \rightarrow 0)$,

$$
\begin{aligned}
& 2 \cos \frac{\theta}{2}=2 \cos \left(\frac{\pi}{3}-\frac{\epsilon}{2}\right)=2\left(\cos \frac{\pi}{3} * \cos \frac{\epsilon}{2}+\sin \frac{\pi}{3} * \sin \frac{\epsilon}{2}\right) \text {, then } \\
& =\cos \frac{\epsilon}{2}+\sqrt{3} \sin \frac{\epsilon}{2} \rightarrow 1+\frac{\sqrt{3}}{2} \epsilon \\
& \frac{1}{n}=\log _{2} 2 \cos \frac{\theta}{2} \rightarrow \log _{2}\left(1+\frac{\sqrt{3}}{2} \epsilon\right), \frac{1}{n} \rightarrow \frac{\sqrt{3}}{2 \ln 2} \epsilon \rightarrow 0 \text {, and } \frac{1}{n}>0, n \rightarrow+\infty \\
& \text { 2) When } \theta=\frac{2 \pi}{3}+\epsilon,(\epsilon>0 \text { and } \epsilon \rightarrow 0) \text {, } \\
& 2 \cos \frac{\theta}{2}=2 \cos \left(\frac{\pi}{3}+\frac{\epsilon}{2}\right)=2\left(\cos \frac{\pi}{3} * \cos \frac{\epsilon}{2}-\sin \frac{\pi}{3} * \sin \frac{\epsilon}{2}\right) \text {, then } \\
& =\cos \frac{\epsilon}{2}-\sqrt{3} \sin \frac{\epsilon}{2} \rightarrow 1-\frac{\sqrt{3}}{2} \epsilon \\
& \frac{1}{n}=\log _{2} 2 \cos \frac{\theta}{2} \rightarrow \log _{2}\left(1-\frac{\sqrt{3}}{2} \epsilon\right), \frac{1}{n} \rightarrow\left(-\frac{\sqrt{3}}{2 \ln 2} \epsilon\right) \rightarrow 0, \text { and } \frac{1}{n}<0, n \rightarrow-\infty
\end{aligned}
$$




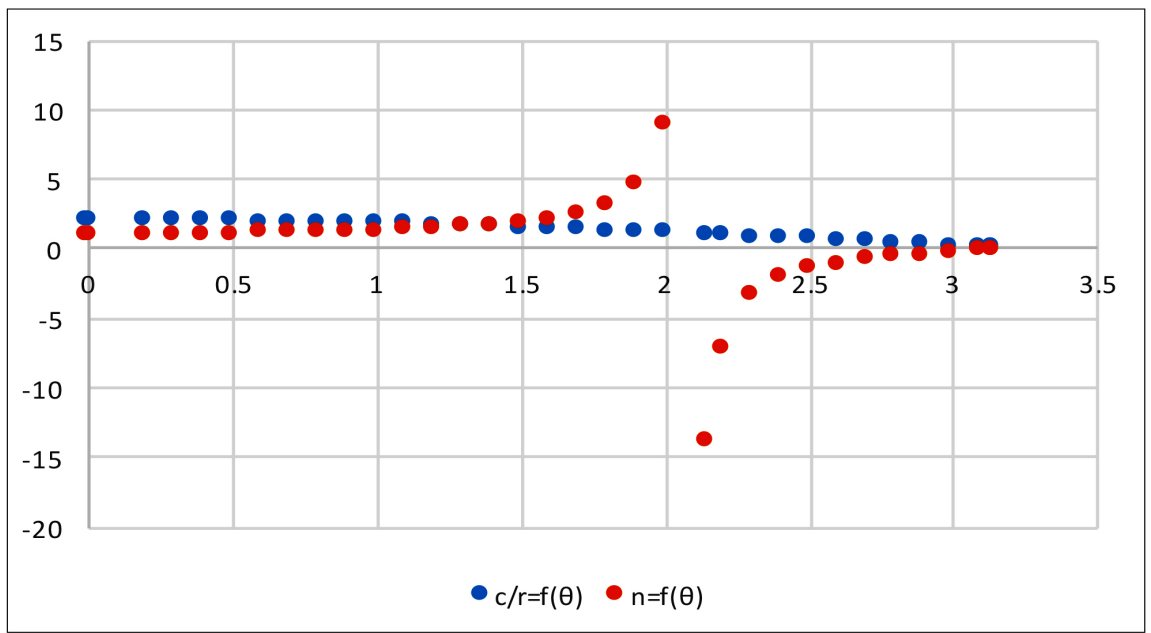

Figure 2. The relationship between $n, \theta$ and $c(a=b)$.

3) When $\theta=\pi-\epsilon,(\epsilon>0$ and $\epsilon \rightarrow 0)$,

$$
\begin{aligned}
2 \cos \frac{\theta}{2} & =2 \cos \left(\frac{\pi}{2}-\frac{\epsilon}{2}\right)=2\left(\cos \frac{\pi}{2} * \cos \frac{\epsilon}{2}+\sin \frac{\pi}{2} * \sin \frac{\epsilon}{2}\right), \text { then } \\
& =0+\sin \frac{\epsilon}{2} \rightarrow+\frac{\epsilon}{2}
\end{aligned}
$$

$\frac{1}{n}=\log _{2} 2 \cos \frac{\theta}{2} \rightarrow \log _{2}\left(\frac{\epsilon}{2}\right) \rightarrow-\infty, n \rightarrow-0$

If $a=b=r$ is integer, $n \in \mathbb{Q}$, then $n=\frac{p}{q}>1,(p, q)=1, p$ and $q$ are positive integer.

$$
2^{\frac{1}{n}}=2^{\frac{q}{p}}=2 \cos \frac{\theta}{2}=\frac{c}{r}
$$

If $c$ can be a positive integer, $2^{\frac{1}{n}}$ must be a rational number. If $2^{\frac{1}{n}}=2^{\frac{q}{p}}$ is rational number, then $2^{\frac{q}{p}}=\frac{t}{s},(t, s)=1, t$ and $s$ are positive integer, $0<\frac{t}{s}<2$.

$$
\begin{aligned}
\left(2^{\frac{q}{p}}\right)^{\frac{p}{q}} & =\left(\frac{t}{s}\right)^{\frac{p}{q}}=2 \\
(t)^{\frac{p}{q}} & =2(s)^{\frac{p}{q}} \\
\left((t)^{\frac{p}{q}}\right)^{q} & =\left(2(s)^{\frac{p}{q}}\right)^{q} \\
(t)^{p} & =2^{q}(s)^{p}
\end{aligned}
$$

$t, s, p, q$ are positive integer, so $2^{q}$ is an even number, then $t$ is an even number, let $t=2 k$ ( $k$ is positive integer),

$$
\begin{aligned}
& (2 k)^{p}=2^{q}(s)^{p} \\
& 2^{(p-q)} k^{p}=s^{p}
\end{aligned}
$$


$n=\frac{p}{q}>1, \quad p>q, p, q$ are positive integer, then $(p-q)>0$ and $(p-q)$ is positive integer, so $2^{p-q}$ is even number, and $(s)^{p}$ is also an even number, then $s$ must be an even number.

Above all, we can prove that $t$ and $s$ are even number, which is contradictory with $(t, s)=1$, therefore, $2^{\frac{1}{n}}=2^{\frac{q}{p}}=\frac{c}{r}$ is irrational number, $r$ is integer, then $c$ must be irrational number.

In summary, $a, b$ and $c$ are the 3 sides of triangle, if $a=b=r$ is positive integer, $n$ is rational number $(n \geq 1)$, and $a, b, c$ satisfy the function $a^{n}+b^{n}=c^{n} \quad(1)$, only if $n=1, c$ can be integer, the relationship between $n$ and $\theta$ is:

$$
\frac{1}{n}=\log _{2}\left(2 \cos \frac{\theta}{2}\right)=\log _{2}\left(\frac{c}{r}\right)
$$

\subsection{The Proof of Fermat's Last Theorem}

The Fermat's Last Theorem is:

$$
a^{n}+b^{n}=c^{n}
$$

When $n$ is integer and $n>2$, the function (1) has no positive integer solution, which means $a, b$ and $c$ can't be positive integer at the same time or when $a, b$, and $c$ is positive integer, $n$ is integer and $n>2$, the function $a^{n}+b^{n}=c^{n}$ cannot be established.

First of all, we have to prove the value of $a, b, c$ in the function (1) can form a triangle.

If $\mathrm{n}$ is integer and $n>2, a, b$ and $c$ are more than 0 , then:

$$
\begin{aligned}
& a^{n}+b^{n}=c^{n}>a^{n}, \text { so } c>a ; \\
& a^{n}+b^{n}=c^{n}>b^{n}, \text { so } c>b ; \\
& a^{n}+b^{n}=c^{n}<(a+b)^{n}, \text { so } c<a+b
\end{aligned}
$$

Therefore, $a, b$, and $c$ can certainly form a triangle $\triangle \mathrm{OAB}[7]$, and the triangle $\triangle \mathrm{OAB}$ is shown in Figure 3.

In the section of 1.1, we have proved that the value of $c$ in the function (1) is irrational even if $n$ is rational $(n>1)$ when $a=b=r$ and $r$ is positive integer. To prove the Fermat's Last Theorem, we have to prove another condition: if $a, b$ and $c$ is positive integer, and $a \neq b$, the function $a^{n}+b^{n}=c^{n}$ (2) can not be established when $n$ is integer and $n>2$.

Similarly, we can also construct the geometric method as same as the section of 2.1. The geometric graph is shown in Figure 3. As shown in Figure 3, OA = $a, \mathrm{OB}=b$, and let $a>b$, the point of $\mathrm{B}$ can move to $\mathrm{B}$ ' on the circle which centered on point $\mathrm{O}$ and the radius of the circle is $b$. Connect point $\mathrm{O}, \mathrm{A}$ and $\mathrm{B}$ to form a triangle $\triangle \mathrm{OAB}$, the angle of $\angle \mathrm{BOB}^{\prime}=\theta, \mathrm{AB}^{\prime}=c, a, b$ and $c$ are the length of the 3 sides of $\triangle \mathrm{OAB}$, so their value are more than 0 .

So $\mathrm{AB}^{\prime 2}=c^{2}=(a+b \cos \theta)^{2}+(b \sin \theta)^{2}=a^{2}+b^{2}+2 a b \cos \theta$ 


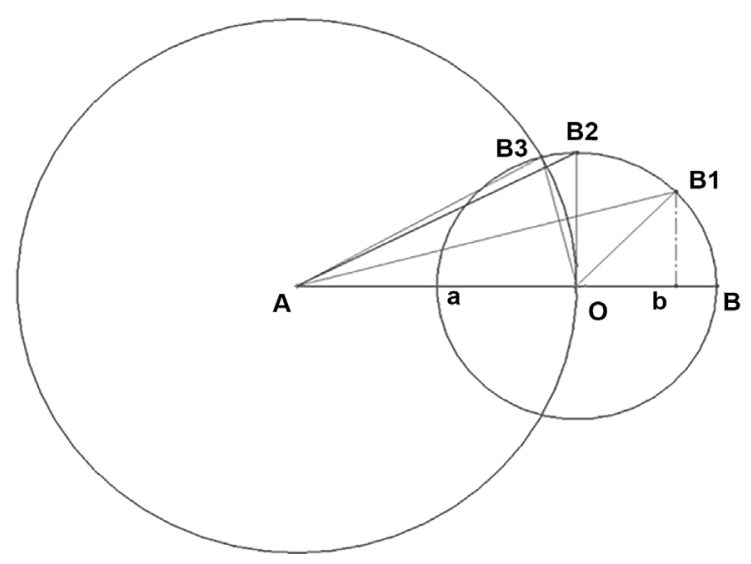

Figure 3. The Geometric construction to prove Fermat's Last Theorem $(a \neq b$ and let $a>b)$.

If $\theta=0, c^{2}=a^{2}+b^{2}+2 a b \cos \theta=a^{2}+b^{2}+2 a b=(a+b)^{2}, \quad c=a+b, n=1$.

If $\theta=\frac{\pi}{2}, c^{2}=a^{2}+b^{2}+2 a b \cos \theta=a^{2}+b^{2}, n=2$, and the value of $a, b, c$ can be integer.

If $\theta \neq \frac{\pi}{2}$ and $\theta \neq 0, a$ and $b$ are positive integer, $a, b$ and $c$ satisfy the function (1), then:

$$
\begin{aligned}
& a^{n}+b^{n}=c^{n}=\left(a^{2}+b^{2}+2 a b \cos \theta\right)^{\frac{n}{2}} \\
& c=\sqrt{a^{2}+b^{2}+2 a b \cos \theta}=\sqrt[n]{a^{n}+b^{n}}
\end{aligned}
$$

Function (7) shows the relationship between $a, b, n$ and $\theta$, what's more, function (7) is the necessary and sufficient conditions of function (1), so:

$$
\begin{gathered}
c=\sqrt{a^{2}+b^{2}+2 a b \cos \theta}=\sqrt{a^{2}+b^{2}+2 a b-2 a b+2 a b \cos \theta} \\
c=\sqrt{(a+b)^{2}-2 a b(1-\cos \theta)}
\end{gathered}
$$

if $c$ is integer, according to function (8), $c<(a+b)$, let $c=(a+t), t$ is integer and $0<t<b$.

$$
\begin{gathered}
c^{2}=(a+t)^{2}=(a+b)^{2}-2 a b(1-\cos \theta) \\
(2 a+b+t)(b-t)=2 a b(1-\cos \theta)
\end{gathered}
$$

$a, b$ and $t$ is integer, so $(2 a+b+t)(b-t)$ is integer, then $\cos \theta$ must be rational. Let $\cos \theta=R$, and $R$ is rational.

1) When $\theta \in\left(0, \frac{\pi}{2}\right), \quad R \in(1,0)$

$$
\begin{gathered}
\left(a^{n}+b^{n}\right)^{2}=a^{2 n}+2 a^{n} b^{n}+b^{2 n} \\
\left(c^{n}\right)^{2}=\left(a^{2}+b^{2}+2 a b \cos \theta\right)^{n}>\left(a^{2}+b^{2}\right)^{n} \\
=a^{2 n}+C_{n}^{1} a^{2(n-1)} b^{2 \times 1}+C_{n}^{2} a^{2(n-2)} b^{2 \times 2}+\cdots+C_{n}^{n} a^{2(n-n)} b^{2 \times n} \\
\text { So } a^{2 n}+2 a^{n} b^{n}+b^{2 n}<\left(a^{2}+b^{2}\right)^{n}<c^{2 n}, a^{n}+b^{n}<c^{n} \quad \text { (12). }
\end{gathered}
$$


Therefore, when $\theta \in\left(0, \frac{\pi}{2}\right), R \in(1,0), c=\sqrt{a^{2}+2 R a b+b^{2}}$ and $c$ can be integer, $n$ is integer and $n>2$, if $a, b$ is positive integer, the relationship between $a, b, c$ and $n$ must be $a^{n}+b^{n}<c^{n}$, no matter $c$ is integer or not.

2) When $\theta \in\left(\frac{\pi}{2}, \pi\right], R \in(0,-1)$

Because of $a^{n}+b^{n}=c^{n}, n$ is integer and $n>2$, so that $c>a$ and $c>b$. However, if $B^{\prime}=B_{3}\left(B_{3}\right.$ is the intersection of the two circles in Figure 3$)$,

$\theta=\angle \mathrm{BOB}_{3}=\frac{\pi}{2}+\arcsin \frac{b}{2 a} \quad$ (please see Figure 3.), it means $\mathrm{AB}^{\prime}=\mathrm{AB}_{3}=\mathrm{AO}=c=a$, and if $\theta=\angle \mathrm{BOB}^{\prime}>\angle \mathrm{BOB}_{3}, \quad \mathrm{AB}^{\prime}=c<a \quad$ (According to Euclid's Elements, big angle to big side) [7], so if $\theta \in\left(\frac{\pi}{2}+\arcsin \frac{b}{2 a}, \pi\right]$, the value of $c<a$.

Therefore, if $\theta \in\left(\frac{\pi}{2}, \frac{\pi}{2}+\arcsin \frac{b}{2 a}\right), R \in\left(-\frac{b}{2 a}, 0\right)$, then $c>a$, and there is possible to make the function $a^{n}+b^{n}=c^{n}$ to be tenable when $n>2$. Let $c=(a+t), t$ is integer and $0<t<b, a \geq b+1$, then

$$
\begin{gathered}
a^{n}+b^{n}=c^{n}=(a+t)^{n} \geq(a+1)^{n} \\
(a+1)^{n}=a^{n}+C_{n}^{1} a^{(n-1)}+C_{n}^{2} a^{(n-2)}+\cdots+1
\end{gathered}
$$

So, if the function $a^{n}+b^{n}=c^{n}$ is established when $n$ is integer and $n>2$, $a>b$, we can get:

$$
\begin{gathered}
a^{n}+b^{n} \geq a^{n}+C_{n}^{1} a^{(n-1)}+C_{n}^{2} a^{(n-2)}+\cdots+C_{n}^{(n-1)} a^{1}+1 \\
1 \geq \frac{C_{n}^{1} a^{(n-1)}+C_{n}^{2} a^{(n-2)}+\cdots+1}{b^{n}} \geq \frac{C_{n}^{1}(b+1)^{(n-1)}+C_{n}^{2}(b+1)^{(n-2)}+\cdots+1}{b^{n}}
\end{gathered}
$$

If $b=1$, according to function (15), $1 \geq C_{n}^{1} a^{(n-1)}+C_{n}^{2} a^{(n-2)}+\cdots+1$ can't be established; if $b>1$, according to function (16), there are two conditions that need to be discussed:

1) $n \geq b+1$, then

$$
\begin{aligned}
& \frac{C_{n}^{1}(b+1)^{(n-1)}+C_{n}^{2}(b+1)^{(n-2)}+\cdots+1}{b^{n}} \\
& >\frac{(b+1)(b+1)^{(n-1)}+(b+1)(b+1)^{(n-2)}+\cdots+(b+1)(b+1)^{1}+b+1+1-b-1}{b^{n}} \\
& =\left(\frac{(b+1)^{n+1}-1}{(b+1)-1}-(b+1)\right) \times \frac{1}{b^{n}} \\
& =\frac{(b+1)^{n+1}-1-b(b+1)}{b^{n+1}}>1
\end{aligned}
$$

(which is contradictory with function (16))

Therefore, if $b>1$ and $n \geq b+1$, the relationship between $a, b, c$ and $n$ must be $a^{n}+b^{n}<c^{n}$, the function (16) can't be satisfied.

2) $2<n<b+1$, then 


$$
\begin{gathered}
a^{n}+b^{n}=c^{n}=(a+t)^{n} \\
a^{n}+b^{n}=a^{n}+C_{n}^{1} a^{(n-1)} t+C_{n}^{2} a^{(n-2)} t^{2}+\cdots+t^{n} \\
t^{n}+C_{n}^{(n-1)} a^{1} t^{(n-1)}+\cdots+C_{n}^{2} a^{(n-2)} t^{2}+C_{n}^{1} a^{(n-1)} t-b^{n}=0
\end{gathered}
$$

If there exists a positive integer $c$ to satisfy the function (17), then $t$ must be a integer, and the value of $t$ is the solution for the equation of degree $n$ with one unknown in function (18), and the solution of $t$ in function (18) is also the solution of $t$ in function (17).

Obviously, one of the solution of $t$ in function (17) must be:

$$
\begin{gathered}
t=-a \pm \sqrt[n]{a^{n}+b^{n}} \text { (if } n \text { is even number) } \\
t=-a+\sqrt[n]{a^{n}+b^{n}} \text { (if } n \text { is odd number) }
\end{gathered}
$$

In this paper, we only discuss the condition of $0<t<b$, so the value of $t$ satisfies the requirement is:

$$
t=-a+\sqrt[n]{a^{n}+b^{n}}
$$

Therefore, we have found a solution for the equation of degree $n$ with one unknown in function (18), and the solution of $t$ is the function (19) and (20).

For example: let $a=2, b=1$, and $n=5$, so the function (18) is equal to the function shows below:

$$
t^{5}+10 t^{4}+40 t^{3}+80 t^{2}+80 t-1=0
$$

According to function (20), we can easily get the solution of $t$ in function (22) is

$$
t=-2+\sqrt[5]{2^{5}+1^{5}}=-2+\sqrt[5]{33}
$$

This is a special example of the solution for the equation of degree $n$ with one unknown. However, how to find the solution for the arbitrarily equation of degree $n$ with one unknown as function (24):

$$
A_{n} t^{n}+A_{(n-1)} t^{(n-1)}+\cdots+A_{2} t^{2}+A_{1} t-B=0
$$

Let's discuss the question in the next Section 2.3.

According to function (18), we can transform it to the function (25):

$$
t^{n-1}+C_{n}^{(n-1)} a^{1} t^{(n-2)}+\cdots+C_{n}^{2} a^{(n-2)} t^{1}+C_{n}^{1} a^{(n-1)}=\frac{b^{n}}{t}
$$

$t$ is a positive integer, so the left side of the equal sign in function (25) must be a positive integer, therefore, the value of $\frac{b^{n}}{t}$ must be a positive integer.

Let the value of $\frac{b^{n}}{t}=m$, and $m$ is a positive integer, then

$$
t^{n-1}+C_{n}^{(n-1)} a^{1} t^{(n-2)}+\cdots+C_{n}^{2} a^{(n-2)} t^{1}+C_{n}^{1} a^{(n-1)}-m=0
$$

Therefore, we have to find the value of $t$ in the function (26), and determine if the value of $t$ can be an integer. If $t$ can't be a positive integer when $n \geq 3$, then the Fermat's last Theorem is right. 
If $n=1$, the function (26) is equal to $1-m=0$, then $\frac{b^{1}}{t}=1, t=b$, so $c=a+t=a+b ;$

If $n=2$, the function (26) is equal to $t+2 a-m=0$, then $\frac{b^{2}}{t}=t+2 a$, $t^{2}+2 a t=b^{2}$, so $a^{2}+b^{2}=a^{2}+t^{2}+2 a t=(a+t)^{2}=c^{2}$.

Therefore, if $n=1$ and $n=2$, the function $a^{n}+b^{n}=c^{n}$ can have integer solution.

If $n=3$, the function (26) is equal to $t^{2}+3 a t+3 a^{2}-m=0$, then $t=\frac{-3 a \pm \sqrt{4 m-3 a^{2}}}{2}$,

The value of $t$ must be integer and $0<t<b$, then $4 m-3 a^{2}=R^{2} a^{2}, R^{2}$ is a positive integer, thus, $4 m=K a^{2}, K-3=R^{2}, K=4,7,12,19, \cdots$, $R=1,2,3,4, \cdots$, then:

$$
\begin{gathered}
t=\frac{-3 a+\sqrt{4 m-3 a^{2}}}{2}=\frac{(R-3) a}{2} \\
\frac{b^{3}}{t}=m=\frac{K a^{2}}{4}=\frac{\left(R^{2}+3\right) a^{2}}{4} \\
b^{3}=\frac{\left(R^{2}+3\right) a^{2}}{4} \times \frac{(R-3) a}{2}=\frac{\left(R^{2}+3\right)(R-3) a^{3}}{8} \\
b=\frac{a}{2} \sqrt[3]{\left(R^{2}+3\right)(R-3)}
\end{gathered}
$$

Therefore, $\left(R^{2}+3\right)(R-3)$ must be a positive cubic number, so $R>3$. Let $\left(R^{2}+3\right)(R-3)=(R-\alpha)^{3}, \alpha$ is positive integer, then:

$$
R^{3}-3 R^{2}+3 R-9=R^{3}-3 \alpha R^{2}+3 \alpha^{2} R-\alpha^{3}
$$

If $\alpha=1, R^{3}-3 \alpha R^{2}+3 \alpha^{2} R-\alpha^{3}=R^{3}-3 R^{2}+3 R-1>R^{3}-3 R^{2}+3 R-9$

$$
R^{3}-3 \alpha R^{2}+3 \alpha^{2} R-\alpha^{3}-\left(R^{3}-3 R^{2}+3 R-9\right)
$$

If $\alpha=2$,

$$
=-3 R^{2}+9 R+1=\Delta=-3\left(R-\frac{3}{2}\right)^{2}+\frac{31}{4}
$$

Because $R$ is positive integer and $R>3, \Delta<0$, so, $R^{3}-6 R^{2}+12 R-8<R^{3}-3 R^{2}+3 R-9$ If $\alpha=3$,

$$
R^{3}-3 \alpha R^{2}+3 \alpha^{2} R-\alpha^{3}-\left(R^{3}-3 R^{2}+3 R-9\right)
$$

$$
=-6 R^{2}+24 R-18=\Delta=-6\left[(R-2)^{2}-1\right]
$$

integer and $R>3, \Delta<0$, so, $R^{3}-9 R^{2}+27 R-27<R^{3}-3 R^{2}+3 R-9$

If $\alpha=\alpha$, and $\alpha>3$, then,

$$
\begin{aligned}
& R^{3}-3 \alpha R^{2}+3 \alpha^{2} R-\alpha^{3}-\left(R^{3}-3 R^{2}+3 R-9\right) \\
& =-(3 \alpha-3) R^{2}+\left(3 \alpha^{2}-3\right) R-\left(\alpha^{3}-9\right) \\
& =-(3 \alpha-3)\left[R^{2}-(\alpha+1) R\right]-\left(\alpha^{3}-9\right) \\
& =\Delta=-(3 \alpha-3)\left[R-\left(\frac{\alpha+1}{2}\right)\right]^{2}+\frac{-\alpha^{3}+3 \alpha^{2}-3 \alpha+33}{4}
\end{aligned}
$$


When $\alpha=4, \frac{-\alpha^{3}+3 \alpha^{2}-3 \alpha+33}{4}=\frac{5}{4}$, Because $R$ is positive integer and $R>3, \Delta<0$, so, $R^{3}-3 \alpha R^{2}+3 \alpha^{2} R-\alpha^{3}<R^{3}-3 R^{2}+3 R-9$

When $\alpha=5, \frac{-\alpha^{3}+3 \alpha^{2}-3 \alpha+33}{4}=-8$, No matter what the value of $R$ is, $R^{3}-3 \alpha R^{2}+3 \alpha^{2} R-\alpha^{3}<R^{3}-3 R^{2}+3 R-9$

The function $f(\alpha)=-\alpha^{3}+3 \alpha^{2}-3 \alpha+33$ is monotonically decreasing function when $\alpha>5$, so $f(\alpha)<-8$ when $\alpha>5$, therefore, No matter what the value of $R$ is, $R^{3}-3 \alpha R^{2}+3 \alpha^{2} R-\alpha^{3}<R^{3}-3 R^{2}+3 R-9$.

Thus, $\left(R^{2}+3\right)(R-3)=(R-\alpha)^{3}$ can't be established when $\alpha$ is positive integer, and $\left(R^{2}+3\right)(R-3)$ can't be a positive cubic number, therefore, the value of $b$ can't be a positive integer.

In conclusion, if $n=3$, and the solution of $t$ in function (26) is positive integer, but the value of $b$ can't be a positive integer, thus, the Fermat's Last Theorem is established when $n=3$.

\subsection{The Solution for the Equation of Degree $\boldsymbol{n}$ with One Unknown}

How to find the solution for the arbitrarily equation of degree $n$ with one unknown as function (24):

$$
A_{n} t^{n}+A_{(n-1)} t^{(n-1)}+\cdots+A_{2} t^{2}+A_{1} t-B=0
$$

This paper only discusses the real solution of function (24). As we already known, the solution of $t$ in the function (18) is

$$
\begin{gathered}
t=-a \pm \sqrt[n]{a^{n}+b^{n}} \text { (if } n \text { is even number) } \\
t=-a+\sqrt[n]{a^{n}+b^{n}} \text { (if } n \text { is odd number) }
\end{gathered}
$$

So, the function (24) can be deformed as function (27):

$$
\begin{aligned}
& A_{n}\left(t^{n}+C_{n}^{(n-1)} a^{1} t^{(n-1)}+\cdots+C_{n}^{2} a^{(n-2)} t^{2}+C_{n}^{1} a^{(n-1)} t-b_{n}\right) \\
& +B_{(n-1)}\left(t^{(n-1)}+C_{(n-1)}^{(n-2)} a^{1} t^{(n-2)}+\cdots+C_{(n-1)}^{2} a^{(n-2)} t^{2}+C_{(n-1)}^{1} a^{(n-1)} t-b_{(n-1)}\right) \\
& +B_{(n-2)}\left(t^{(n-2)}+C_{(n-2)}^{(n-3)} a^{1} t^{(n-3)}+\cdots+C_{(n-2)}^{2} a^{(n-4)} t^{2}+C_{(n-2)}^{1} a^{(n-3)} t-b_{(n-2)}\right) \\
& +\cdots+B_{2}\left(t^{2}+C_{2}^{1} a^{1} t^{1}-b_{2}\right)+B_{1}\left(t-b_{1}\right)=0
\end{aligned}
$$

The coefficients in function (27) are shown below:

$$
\begin{aligned}
& B_{(n-1)}=\left(A_{(n-1)}-A_{n} C_{n}^{(n-1)} a^{1}\right) \\
& B_{(n-2)}=\left[A_{(n-2)}-A_{n} C_{n}^{(n-2)} a^{2}-B_{(n-1)} C_{(n-1)}^{(n-2)} a^{1}\right] \\
& \quad=\left[A_{(n-2)}-A_{n} C_{n}^{(n-2)} a^{2}-\left(A_{(n-1)}-A_{n} C_{n}^{(n-1)} a^{1}\right) C_{(n-1)}^{(n-2)} a^{1}\right] \\
& \vdots \\
& B_{2}=\left[A_{2}-A_{n} C_{n}^{2} a^{(n-2)}-B_{(n-1)} C_{(n-1)}^{2} a^{(n-2)}-B_{(n-2)} C_{(n-2)}^{2} a^{(n-4)}-\cdots-B_{3} C_{3}^{2} a^{1}\right] \\
& B_{1}=\left[A_{1}-A_{n} C_{n}^{1} a^{(n-1)}-B_{(n-1)} C_{(n-1)}^{1} a^{(n-1)}-B_{(n-2)} C_{(n-2)}^{1} a^{(n-3)}-\cdots-B_{2} C_{2}^{1} a^{1}\right]
\end{aligned}
$$




$$
A_{n} b_{n}+B_{(n-1)} b_{(n-1)}+B_{(n-2)} b_{(n-2)}+\cdots+B_{2} b_{2}+B_{1} b_{1}=B
$$

The solution of $t$ in the function (27) is $t(k)$, and the value $t(k)$ after substituting the equation coefficient with $A_{n}, B_{(n-1)}, B_{(n-2)}, \cdots, B_{2}$ and $B_{1}$ in the function (27) is zero. According to the function (19) and (20), we can easily get the value $t(k)(k=1,2, \cdots, n)$ are:

As $0<t<b$, so:

$$
\begin{aligned}
& t(n)=-a+\sqrt[n]{a^{n}+b_{n}} \\
& t(n-1)=-a+\sqrt[(n-1)]{a^{(n-1)}+b_{(n-1)}} \\
& t(n-2)=-a+\sqrt[(n-2)]{a^{(n-2)}+b_{(n-2)}} \\
& \vdots \\
& t(2)=-a+\sqrt[2]{a^{2}+b_{2}} \\
& t(1)=b_{1}
\end{aligned}
$$

If we want the function (27) to be established, then all the value of $t(k)$ must be equal, and we can find the solution for the equation of degree $n$ with one unknown. We can set the value of $a$ and $a \neq 0$, so here are $n$ equations and we have to find the solution of $b_{1}, b_{2}, \cdots, b_{n}$, and the solution can be found.

1) Part one: let's find the solution for the random equation of degree 3 with one unknown, the equation is shown below:

$$
A_{3} t^{3}+A_{2} t^{2}+A_{1} t-B=0
$$

The function (29) can be deformed as:

$$
A_{3}\left(t^{3}+3 a t^{2}+3 a^{2} t-b_{3}\right)+\left(A_{1}-A_{3} \times 3 a^{2}\right) t-\left(B-A_{3} b_{3}\right)=0
$$

and $A_{3} \times 3 a=A_{2}, \quad a=\frac{A_{2}}{3 A_{3}}$

we can easily get the value $t(3)$ and $t(1)$ :

$$
\left\{\begin{array}{l}
t(3)=-\frac{A_{2}}{3 A_{3}}+\sqrt[3]{\left(\frac{A_{2}}{3 A_{3}}\right)^{3}+b_{3}} \\
t(1)=\frac{B-A_{3} b_{3}}{A_{1}-\frac{A_{2}^{2}}{3 A_{3}}}
\end{array}\right.
$$

In order to make the function (29) to be established, the value of $t(3)$ and $t(1)$ must be equal, let $t(1)=t(3)=m$, then:

$$
b_{3}=\frac{B-m\left(A_{1}-\frac{A_{2}^{2}}{3 A_{3}}\right)}{A_{3}}
$$




$$
\begin{gathered}
\left(m+\frac{A_{2}}{3 A_{3}}\right)^{3}=\left(\frac{A_{2}}{3 A_{3}}\right)^{3}+b_{3}=\left(\frac{A_{2}}{3 A_{3}}\right)^{3}+\frac{B-m\left(A_{1}-\frac{A_{2}^{2}}{3 A_{3}}\right)}{A_{3}} \\
\left(m+\frac{A_{2}}{3 A_{3}}\right)^{3}=-\frac{A_{1}-\frac{A_{2}^{2}}{3 A_{3}}}{A_{3}}\left(m+\frac{A_{2}}{3 A_{3}}\right)+\frac{27 A_{3}^{2} B+9 A_{1} A_{2} A_{3}-2 A_{2}^{3}}{27 A_{3}^{3}}
\end{gathered}
$$

Let $m+\frac{A_{2}}{3 A_{3}}=w$, then:

$$
w^{3}+\frac{3 A_{1} A_{3}-A_{2}^{2}}{3 A_{3}^{2}} w-\frac{27 A_{3}^{2} B+9 A_{1} A_{2} A_{3}-2 A_{2}^{3}}{27 A_{3}^{3}}=0
$$

According to the Cardano Formula of the General Solution of Cubic Algebraic Equations [8], the real solution of function (31) is:

$$
\begin{gathered}
w=\sqrt[3]{-\frac{q}{2}+\sqrt{\left(\frac{q}{2}\right)^{2}+\left(\frac{p}{3}\right)^{3}}}+\sqrt[3]{-\frac{q}{2}-\sqrt{\left(\frac{q}{2}\right)^{2}+\left(\frac{p}{3}\right)^{3}}} \\
\left\{\begin{array}{l}
p=\frac{3 A_{1} A_{3}-A_{2}^{2}}{3 A_{3}^{2}} \\
q=-\frac{27 A_{3}^{2} B+9 A_{1} A_{2} A_{3}-2 A_{2}^{3}}{27 A_{3}^{3}}
\end{array}\right.
\end{gathered}
$$

Therefore, the solution of $t$ satisfy the function in (29) is $t=-\frac{A_{2}}{3 A_{3}}+w$

For example, Let's find the solution of $\mathrm{t}$ in the function (33) below:

$$
\begin{gathered}
2 t^{3}+3 t^{2}+4 t-5=0 \\
p=\frac{3 A_{1} A_{3}-A_{2}^{2}}{3 A_{3}^{2}}=\frac{5}{4} \\
q=-\frac{27 A_{3}^{2} B+9 A_{1} A_{2} A_{3}-2 A_{2}^{3}}{27 A_{3}^{3}}=-\frac{13}{4} \\
w=\sqrt[3]{\frac{13}{8}+\sqrt{\left(\frac{13}{8}\right)^{2}+\left(\frac{5}{12}\right)^{3}}}+\sqrt[3]{\frac{13}{8}-\sqrt{\left(\frac{13}{8}\right)^{2}+\left(\frac{5}{12}\right)^{3}}} \approx 1.20393969
\end{gathered}
$$

so the real solution of $t$ in the function (33) is $t=-\frac{1}{2}+w \approx 0.70393969$

If $n=4$, the function (26) is equal to the function (34) shows below:

$$
\begin{gathered}
t^{3}+4 a^{1} t^{2}+6 a^{2} t^{1}-\left(m-4 a^{3}\right)=0 \\
p=\frac{3 A_{1} A_{3}-A_{2}^{2}}{3 A_{3}^{2}}=\frac{2 a^{2}}{3} \\
q=-\frac{27 A_{3}^{2} B+9 A_{1} A_{2} A_{3}-2 A_{2}^{3}}{27 A_{3}^{3}}=\frac{20 a^{3}}{27}-m
\end{gathered}
$$




$$
\begin{gathered}
w=\sqrt[3]{-\frac{q}{2}+\sqrt{\left(\frac{q}{2}\right)^{2}+\left(\frac{p}{3}\right)^{3}}}+\sqrt[3]{-\frac{q}{2}-\sqrt{\left(\frac{q}{2}\right)^{2}+\left(\frac{p}{3}\right)^{3}}} \\
u=\sqrt{\left(\frac{q}{2}\right)^{2}+\left(\frac{p}{3}\right)^{3}}=\sqrt{\left(\frac{10 a^{3}}{27}-\frac{m}{2}\right)^{2}+\left(\frac{2 a^{2}}{9}\right)^{3}}
\end{gathered}
$$

The value of $u$ in the function (35) must be a integer, therefore, $\left(\frac{10 a^{3}}{27}-\frac{m}{2}\right)^{2}+\left(\frac{2 a^{2}}{9}\right)^{3}$ must be a positive square integer, otherwise, the value of $w$ can't be a rational number.

Let $m=2 k, a=3 s, k$ and $s$ is positive integer, then:

$$
u=\sqrt{\left(\frac{10 a^{3}}{27}-\frac{m}{2}\right)^{2}+\left(\frac{2 a^{2}}{9}\right)^{3}}=\sqrt{\left(10 s^{3}-k\right)^{2}+\left(2 s^{2}\right)^{3}}
$$

Obviously, if $\left(10 s^{3}-k\right)^{2}=K^{2} s^{6}, K^{2}+8=R^{2}, K$ and $R$ is a positive integer, under this condition, the value of $u$ can be a integer. Then, $K^{2}=1,8,17,28, \cdots$, $R=3,4,5,6, \cdots$, and $u= \pm R s^{3}$.However, the value of $K$ must be a positive square integer, a positive square integer plus 8 is still a square integer, only when $K^{2}=1, R^{2}=9$ can satisfy the requirement, then $k=9 s^{3}$ or $11 s^{3}, u= \pm 3 s^{3}$.

$$
\begin{gathered}
p=\frac{2 a^{2}}{3}=6 s^{2} \\
q=-\frac{27 A_{3}^{2} B+9 A_{1} A_{2} A_{3}-2 A_{2}^{3}}{27 A_{3}^{3}}=\frac{20 a^{3}}{27}-m=20 s^{3}-2 k= \pm 2 s^{3}
\end{gathered}
$$

When $k=9 s^{3}$

$$
w=\sqrt[3]{s^{3}+\left( \pm 3 s^{3}\right)}+\sqrt[3]{s^{3}-\left( \pm 3 s^{3}\right)}=\sqrt[3]{4} s+\sqrt[3]{-2} s
$$

When $k=11 s^{3}$

$$
w=\sqrt[3]{-s^{3}+\left( \pm 3 s^{3}\right)}+\sqrt[3]{-s^{3}-\left( \pm 3 s^{3}\right)}=\sqrt[3]{2} s+\sqrt[3]{-4} s
$$

Absolutely, the value of $w$ is irrational, therefore, the solution of $t$ satisfy the function in (34) is $t=-12 s+w$ is irrational.

In conclusion, if $n=4$, the solution of $t$ in function (26) is irrational, thus, the Fermat's Last Theorem is established when $n=4$.

2) Part two: let's discuss the solution for the random equation of degree 4 with one unknown, the equation is shown below:

$$
A_{4} t^{4}+A_{3} t^{3}+A_{2} t^{2}+A_{1} t-B=0
$$

The function (32) can be deformed as:

$$
\begin{aligned}
& A_{4}\left(t^{4}+4 a t^{3}+6 a^{2} t^{2}+4 a^{3} t-b_{4}\right)+\left(A_{2}-A_{4} \times 6 a^{2}\right) t^{2} \\
& +\left(A_{1}-A_{4} \times 4 a^{3}\right) t-\left(B-A_{4} b_{4}\right)=0
\end{aligned}
$$

and $A_{4} \times 4 a=A_{3}, \quad a=\frac{A_{3}}{4 A_{4}}$ 
we can easily get the value $t(4)$ and $t(2)$ :

$$
\left\{\begin{array}{l}
t(4)=-\frac{A_{3}}{4 A_{4}}+\sqrt[4]{\left(\frac{A_{3}}{4 A_{4}}\right)^{4}+b_{4}} \\
t(2)=\frac{-\left(A_{1}-\frac{A_{3}^{3}}{16 A_{4}^{2}}\right) \pm \sqrt{\left(A_{1}-\frac{A_{3}^{3}}{16 A_{4}^{2}}\right)^{2}+4\left(A_{2}-\frac{3 A_{3}^{2}}{8 A_{4}}\right)\left(B-A_{4} b_{4}\right)}}{2\left(A_{2}-\frac{3 A_{3}^{2}}{8 A_{4}}\right)}
\end{array}\right.
$$

Similarly, in order to make the function (33) to be established, the value of $t(4)$ and $t(2)$ must be equal, let $t(4)=t(2)=m$, then

$$
\begin{gathered}
b_{4}=\frac{B-\left(A_{2}-\frac{3 A_{3}^{2}}{8 A_{4}}\right) m^{2}-\left(A_{1}-\frac{A_{3}^{3}}{16 A_{4}^{2}}\right) m}{A_{4}} \\
\left(m+\frac{A_{3}}{4 A_{4}}\right)^{4}=\left(\frac{A_{3}}{4 A_{4}}\right)^{4}+b_{4} \\
=\left(\frac{A_{3}}{4 A_{4}}\right)^{4}+\frac{B-\left(A_{2}-\frac{3 A_{3}^{2}}{8 A_{4}}\right) m^{2}-\left(A_{1}-\frac{A_{3}^{3}}{16 A_{4}^{2}}\right) m}{A_{4}} \\
\left(m+\frac{A_{3}}{4 A_{4}}\right)^{4}+\frac{8 A_{2} A_{4}-3 A_{3}^{2}}{8 A_{4}^{2}}\left(m+\frac{A_{3}}{4 A_{4}}\right)^{2} \\
-\frac{8 A_{2} A_{3} A_{4}^{2}-3 A_{3}^{3} A_{4}-16 A_{1} A_{4}^{2}+A_{3}^{3}\left(m+\frac{A_{3}}{4 A_{4}}\right)}{16 A_{4}^{3}}\left(128 A_{4}^{3} B+8 A_{2} A_{3}^{2} A_{4}-16 A_{2} A_{3}^{2} A_{4}^{2}+6 A_{3}^{4} A_{4}+32 A_{1} A_{3} A_{4}^{2}-\frac{9}{2} A_{3}^{4}\right. \\
=\frac{128 A_{4}^{4}}{}
\end{gathered}
$$

Let $m+\frac{A_{3}}{4 A_{4}}=w$, then:

$$
\begin{gathered}
w^{4}+p w^{2}+q w+r=0 \\
p=\frac{8 A_{2} A_{4}-3 A_{3}^{2}}{8 A_{4}^{2}} \\
q=-\frac{8 A_{2} A_{3} A_{4}^{2}-3 A_{3}^{3} A_{4}-16 A_{1} A_{4}^{2}+A_{3}^{3}}{16 A_{4}^{3}} \\
r=-\frac{128 A_{4}^{3} B+8 A_{2} A_{3}^{2} A_{4}-16 A_{2} A_{3}^{2} A_{4}^{2}+6 A_{3}^{4} A_{4}+32 A_{1} A_{3} A_{4}^{2}-\frac{9}{2} A_{3}^{4}}{128 A_{4}^{4}}
\end{gathered}
$$

then the function (34) can be transformed to the function (35):

$$
\begin{gathered}
w^{4}+p w^{2}+q w+r=\left(w^{2}+k w+u\right)\left(w^{2}-k w+v\right)=0 \\
\left(w^{2}+k w+u\right)\left(w^{2}-k w+v\right)=w^{4}+\left(u+v-k^{2}\right) w^{2}+k(v-u) w+u v
\end{gathered}
$$


Then we can get:

$$
\left\{\begin{array}{l}
p=u+v-k^{2} \\
q=k(v-u) \\
r=u v
\end{array}\right.
$$

We can set a value for $k$ and $k \neq 0, p, q$, and $r$ are the known number, therefore, we can find the value of $u$ and $v$ are:

$$
\begin{gathered}
u=\frac{k^{3}+p k-q}{2 k} \\
v=\frac{k^{3}+p k+q}{2 k} \\
\frac{k^{3}+p k-q}{2 k} \times \frac{k^{3}+p k+q}{2 k}=r \\
k^{6}+2 p k^{4}+\left(p^{2}-4 r\right) k^{2}-q^{2}=0
\end{gathered}
$$

Let $k^{2}=x$, then the function (36) can be deformed as :

$$
x^{3}+2 p x^{2}+\left(p^{2}-4 r\right) x-q^{2}=0
$$

Now, the problem is transformed to find the solution $x$ for function (37), according to the results of Part one, we can get the solution $x$ is:

$$
\begin{gathered}
W=\sqrt[3]{-\frac{Q}{2}+\sqrt{\left(\frac{Q}{2}\right)^{2}+\left(\frac{P}{3}\right)^{3}}}+\sqrt[3]{-\frac{Q}{2}-\sqrt{\left(\frac{Q}{2}\right)^{2}+\left(\frac{P}{3}\right)^{3}}} \\
P=\frac{3\left(p^{2}-4 r\right)-4 p^{2}}{3}=-\frac{p^{2}+12 r}{3} \\
Q=-\frac{27 q^{2}+18\left(p^{2}-4 r\right) p-16 p^{3}}{27}=-\frac{27 q^{2}-72 p r+2 p^{3}}{27}
\end{gathered}
$$

And the solution $x=-\frac{2 p}{3}+W$

$$
\begin{gathered}
k_{0}=k_{1,2}= \pm \sqrt{x}=\sqrt{-\frac{2 p}{3}+W} \\
u_{0}=\frac{k_{0}^{3}+p k_{0}-q}{2 k_{0}} \\
v_{0}=\frac{k_{0}^{3}+p k_{0}+q}{2 k_{0}} \\
\left(w^{2}+k_{0} w+u_{0}\right)\left(w^{2}-k_{0} w+v_{0}\right)=0
\end{gathered}
$$

Then the solutions $w$ in the function (35) are:

$$
\begin{gathered}
w_{1,2}=\frac{-k_{0} \pm \sqrt{k_{0}^{2}-4 u_{0}}}{2} \\
w_{3,4}=\frac{k_{0} \pm \sqrt{k_{0}^{2}-4 v_{0}}}{2}
\end{gathered}
$$


Therefore, the solution of $t$ in the function (32) are:

$$
\begin{aligned}
& t_{1,2}=-\frac{A_{3}}{4 A_{4}}+w_{1,2} \\
& t_{3,4}=-\frac{A_{3}}{4 A_{4}}+w_{3,4}
\end{aligned}
$$

For example, Let's find the solution of $t$ in the function (39) below:

$$
\begin{aligned}
& t^{4}+2 t^{3}+3 t^{2}+4 t-5=0 \\
& p=\frac{8 A_{2} A_{4}-3 A_{3}^{2}}{8 A_{4}^{2}}=\frac{3}{2} \\
& q=-\frac{8 A_{2} A_{3} A_{4}^{2}-3 A_{3}^{3} A_{4}-16 A_{1} A_{4}^{2}+A_{3}^{3}}{16 A_{4}^{3}}=2 \\
& r=-\frac{128 A_{4}^{3} B+8 A_{2} A_{3}^{2} A_{4}-16 A_{2} A_{3}^{2} A_{4}^{2}+6 A_{3}^{4} A_{4}+32 A_{1} A_{3} A_{4}^{2}-\frac{9}{2} A_{3}^{4}}{128 A_{4}^{4}}=-\frac{103}{16} \\
& P=-\frac{p^{2}+12 r}{3}=25 \\
& Q=-\frac{27 q^{2}-72 p r+2 p^{3}}{27}=-30 \\
& W=\sqrt[3]{-\frac{Q}{2}+\sqrt{\left(\frac{Q}{2}\right)^{2}+\left(\frac{P}{3}\right)^{3}}}+\sqrt[3]{-\frac{Q}{2}-\sqrt{\left(\frac{Q}{2}\right)^{2}+\left(\frac{P}{3}\right)^{3}}} \approx 1.14063859 \\
& x=-\frac{2 p}{3}+W \approx-1+1.14063859=0.14063859 \\
& k_{1}=\sqrt{x}=\sqrt{-\frac{2 p}{3}+W} \approx 0.37501813 \\
& u_{1}=\frac{k_{1}^{3}+p k_{1}-q}{2 k_{0}} \approx-1.8462185 \\
& v_{1}=\frac{k_{1}^{3}+p k_{1}+q}{2 k_{0}} \approx 3.48685708 \\
& w_{1,2}=\frac{-k_{1} \pm \sqrt{k_{1}^{2}-4 u_{1}}}{2} \approx 1.18412432 \text { or }-1.5591424 \\
& k_{2}=-\sqrt{x}=\sqrt{-\frac{2 p}{3}+W} \approx-0.37501813 \\
& u_{2}=\frac{k_{2}^{3}+p k_{2}-q}{2 k_{2}} \approx 3.48685708 \\
& v_{2}=\frac{k_{2}^{3}+p k_{2}+q}{2 k_{2}} \approx-1.8462185 \\
& w_{3,4}=\frac{k_{2} \pm \sqrt{k_{2}^{2}-4 u_{2}}}{2}=0.1875091+1.8578744 i \text { or } 0.1875091-1.8578744 i
\end{aligned}
$$


Therefore, the solutions of $t$ in the function (32) are:

$$
\begin{aligned}
& t_{1,2}=-\frac{A_{3}}{4 A_{4}}+w_{1,2} \approx 0.68412432 \text { or }-2.0591424 \\
& t_{3,4}=-\frac{A_{3}}{4 A_{4}}+w_{3,4}=-0.3124909+1.8578744 i \text { or }-0.3124909-1.8578744 i
\end{aligned}
$$

Similarly, if $n=5$, the function (26) is equal to the function (40) shows below:

$$
\begin{aligned}
& t^{4}+5 a t^{3}+10 a^{2} t^{2}+10 a^{3} t^{1}-\left(m-5 a^{4}\right)=0 \\
& p=\frac{8 A_{2} A_{4}-3 A_{3}^{2}}{8 A_{4}^{2}}=\frac{5}{8} a^{2} \\
& q=-\frac{8 A_{2} A_{3} A_{4}^{2}-3 A_{3}^{3} A_{4}-16 A_{1} A_{4}^{2}+A_{3}^{3}}{16 A_{4}^{3}}=\frac{5}{8} a^{3} \\
& r=-\frac{128 A_{4}^{3} B+8 A_{2} A_{3}^{2} A_{4}-16 A_{2} A_{3}^{2} A_{4}^{2}+6 A_{3}^{4} A_{4}+32 A_{1} A_{3} A_{4}^{2}-\frac{9}{2} A_{3}^{4}}{128 A_{4}^{4}}=\frac{205}{256} a^{4}-m \\
& P=-\frac{p^{2}+12 r}{3}=4 m-\frac{10}{3} a^{4} \\
& Q=-\frac{27 q^{2}-72 p r+2 p^{3}}{27}=-\frac{45 m a^{2}-25 a^{6}}{27} \\
& W=\sqrt[3]{-\frac{Q}{2}+\sqrt{\left(\frac{Q}{2}\right)^{2}+\left(\frac{P}{3}\right)^{3}}}+\sqrt[3]{-\frac{Q}{2}-\sqrt{\left(\frac{Q}{2}\right)^{2}+\left(\frac{P}{3}\right)^{3}}} \\
& u=\sqrt{\left(\frac{Q}{2}\right)^{2}+\left(\frac{P}{3}\right)^{3}}=\sqrt{\left(\frac{25 a^{6}}{54}-\frac{45 m a^{2}}{54}\right)^{2}+\left(\frac{4 m}{3}-\frac{10 a^{4}}{9}\right)^{3}}
\end{aligned}
$$

The value of $u$ in the function (41) must be a integer, therefore, $\left(\frac{25 a^{6}}{54}-\frac{45 m a^{2}}{54}\right)^{2}+\left(\frac{4 m}{3}-\frac{10 a^{4}}{9}\right)^{3}$ must be a positive square integer, otherwise, the value of $w$ can't be a rational number.

Let $m=k a^{4}, k$ is positive number, then:

$$
\begin{gathered}
u=\sqrt{\left(\frac{25-45 k}{54}\right)^{2} a^{12}+\left(\frac{12 k-10}{9}\right)^{3} a^{12}}=\sqrt{\alpha^{2}+\beta^{3}} a^{6} \\
-\frac{Q}{2}+u=\left(-\alpha+\sqrt{\alpha^{2}+\beta^{3}}\right) a^{6} \\
W=\sqrt[3]{-\alpha+\sqrt{\alpha^{2}+\beta^{3}}} a^{2}+\sqrt[3]{-\alpha-\sqrt{\alpha^{2}+\beta^{3}}} a^{2}=(K+S) a^{2} \\
x=-\frac{2 p}{3}+W=-\frac{5}{12} a^{2}+(K+S) a^{2} \\
k_{0}= \pm \sqrt{x}= \pm a \sqrt{(K+S)-\frac{5}{12}}= \pm \gamma a
\end{gathered}
$$




$$
\begin{aligned}
u_{0}=\frac{k_{0}^{3}+p k_{0}-q}{2 k_{0}}= & \frac{(K+S)-\frac{5}{12}}{2} a^{2}+\frac{5}{16} a^{2}-\frac{5}{16 \gamma} a^{2} \\
v_{0} & =\frac{k_{0}^{3}+p k_{0}+q}{2 k_{0}} \\
w_{1,2} & =\frac{-k_{0} \pm \sqrt{k_{0}^{2}-4 u_{0}}}{2} \\
w_{3,4} & =\frac{k_{0} \pm \sqrt{k_{0}^{2}-4 u_{0}}}{2} \\
t_{1,2} & =-\frac{A_{3}}{4 A_{4}}+w_{1,2} \\
t_{3,4} & =-\frac{A_{3}}{4 A_{4}}+w_{3,4}
\end{aligned}
$$

Obviously,

$$
\begin{aligned}
k_{0}^{2}-4 u_{0} & =\left[(K+S)-\frac{5}{12}\right] a^{2}-4\left[\frac{(K+S)-\frac{5}{12}}{2} a^{2}+\frac{5}{16} a^{2}-\frac{5}{16 \gamma} a^{2}\right] \\
& =\left[\frac{5}{4 \gamma}-\frac{5}{4}-\gamma^{2}\right] a^{2}
\end{aligned}
$$

must be a square number, then $\frac{a^{2}}{4 \gamma^{2}}\left[5 \gamma-5 \gamma^{2}-4 \gamma^{4}\right]$ must be a square number,

$$
\left[5 \gamma-5 \gamma^{2}-4 \gamma^{4}\right]=\delta^{2}=\left(u \gamma^{2}-v\right)^{2}=v^{2}-2 u v \gamma^{2}+u^{2} \gamma^{4}
$$

Clearly, the function (42) can't be established, therefore, the value of $w$ is irrational, and the solutions of $t$ satisfy the function in (40) is $t=-\frac{5}{4} a+w$ is irrational.

In conclusion, if $n=5$, the solution of $t$ in function (26) is irrational, thus, the Fermat's Last Theorem is established when $n=5$.

3) Part three: let's discuss the solution for the random equation of degree 5 with one unknown, the equation is shown below:

$$
A_{5} t^{5}+A_{4} t^{4}+A_{3} t^{3}+A_{2} t^{2}+A_{1} t-B=0
$$

The function (43) can be deformed as:

$$
\begin{aligned}
& A_{5}\left(t^{5}+5 a t^{4}+10 a^{2} t^{3}+10 a^{3} t^{2}+5 a^{4} t-b_{5}\right)+\left(A_{3}-A_{5} \times 10 a^{2}\right) t^{3} \\
& +\left(A_{2}-A_{5} \times 10 a^{3}\right) t^{2}+\left(A_{1}-A_{5} \times 5 a^{4}\right) t-\left(B-A_{5} b_{5}\right)=0
\end{aligned}
$$

and $A_{5} \times 5 a=A_{4}, \quad a=\frac{A_{4}}{5 A_{5}}$

The function (44) can also be deformed as: 


$$
\begin{aligned}
& A_{5}\left(t^{5}+5 a t^{4}+10 a^{2} t^{3}+10 a^{3} t^{2}+5 a^{4} t-b_{5}\right)+\left(A_{3}-A_{5} \times 10 a^{2}\right)\left(t^{3}+3 c t^{2}+3 c^{2} t-b_{3}\right) \\
& +\left[A_{1}-A_{5} \times 5 a^{4}-\left(A_{3}-A_{5} \times 10 a^{2}\right) \times 3 c^{2}\right] t-\left[B-A_{5} b_{5}-\left(A_{3}-A_{5} \times 10 a^{2}\right) b_{3}\right]=0 \\
& \text { and } a=\frac{A_{4}}{5 A_{5}}, \quad\left(A_{3}-A_{5} \times 10 a^{2}\right) \times 3 c=\left(A_{2}-A_{5} \times 10 a^{3}\right), \quad c=\frac{A_{2}-A_{5} \times 10 a^{3}}{3\left(A_{3}-A_{5} \times 10 a^{2}\right)}
\end{aligned}
$$

We can easily get the value $t(5), t(3)$ and $t(1)$ :

$$
\left\{\begin{array}{l}
t(5)=-a+\sqrt[5]{a^{5}+b_{5}} \\
t(3)=-c+\sqrt[3]{c^{3}+b_{3}} \\
t(1)=\frac{B-A_{5} b_{5}-\left(A_{3}-A_{5} \times 10 a^{2}\right) b_{3}}{A_{1}-A_{5} \times 5 a^{4}-\left(A_{3}-A_{5} \times 10 a^{2}\right) \times 3 c^{2}}
\end{array}\right.
$$

In order to make the function (43) to be established, the value of $t(5), t(3)$ and $t(1)$ must be equal, let $t(5)=t(3)=t(1)=m$, then,

$$
m=\frac{B-A_{5}\left[(m+a)^{5}-a^{5}\right]-\left(A_{3}-A_{5} \times 10 a^{2}\right)\left[(m+c)^{3}-c^{3}\right]}{A_{1}-A_{5} \times 5 a^{4}-\left(A_{3}-A_{5} \times 10 a^{2}\right) \times 3 c^{2}}
$$

The function (45) can be arranged as:

$$
\begin{gathered}
w^{5}+p w^{3}+q w^{2}+r w+s=0 \\
m+a=w
\end{gathered}
$$

$$
\left\{\begin{array}{l}
p=\frac{A_{3}-A_{5} \times 10 a^{2}}{A_{5}} \\
q=\frac{\left(A_{3}-A_{5} \times 10 a^{2}\right) \times 3(c-a)}{A_{5}} \\
r=\frac{\left(A_{3}-A_{5} \times 10 a^{2}\right) \times 3(c-a)^{2}+\left[A_{1}-A_{5} \times 5 a^{4}-\left(A_{3}-A_{5} \times 10 a^{2}\right) \times 3 c^{2}\right]}{A_{5}} \\
s=\frac{\left(A_{3}-A_{5} \times 10 a^{2}\right)(c-a)^{3}-a\left[A_{1}-A_{5} \times 5 a^{4}-\left(A_{3}-A_{5} \times 10 a^{2}\right) \times 3 c^{2}\right]-B-A_{5} a^{5}-\left(A_{3}-A_{5} \times 10 a^{2}\right) c^{3}}{A_{5}}
\end{array}\right.
$$

The function (46) can be deformed as:

$$
w\left(w^{4}+p w^{2}+r\right)+\left(q w^{2}+s\right)=0
$$

Let $w^{2}=x$, then the function (47) can be deformed as:

$$
\begin{aligned}
w\left(x^{2}+p x+r\right)+(q x+s) & =w(q x+s)(\alpha x+\beta)+(q x+s) \\
& =(q x+s)[w(\alpha x+\beta)+1] \\
& =\left(q w^{2}+s\right)\left(\alpha w^{3}+\beta w+1\right)
\end{aligned}
$$

If $p, r, \alpha, \beta$ satisfies the condition (49) below:

$$
\left\{\begin{array}{l}
q \alpha=1 \\
s \beta=r \\
s \alpha+q \beta=s \frac{1}{q}+q \frac{r}{s}=p \\
s^{2}+q^{2} r=p q s
\end{array}\right.
$$


Therefore, if $p, q, r, s$ satisfy the function (49), the solution of the function (48) are easy to be found.

$$
\begin{gathered}
w_{1,2}= \pm \sqrt{-\frac{s}{q}} \\
w_{3,4,5}=w=\sqrt[3]{-\frac{q}{2}+\sqrt{\left(\frac{q}{2}\right)^{2}+\left(\frac{q r}{3 s}\right)^{3}}}+\sqrt[3]{-\frac{q}{2}-\sqrt{\left(\frac{q}{2}\right)^{2}+\left(\frac{q r}{3 s}\right)^{3}}}
\end{gathered}
$$

But, if those conditions can't be satisfied, then the function (46) can't be deformed as function (47), so we can deform the function (46) to function (50):

$$
\left(w^{3}+d w^{2}+e w+f\right)\left(w^{2}-d w+g\right)=0
$$

The unknown number $d, e, f, g$ satisfy the relationship (51) below:

$$
\left\{\begin{array}{l}
e-d^{2}+g=p \\
f-d e+d g=q \\
-d f+e g=r \\
f g=s
\end{array}\right.
$$

There are four functions in (51), so we can solve each value of the unknown number $d, e, f, g$, then the solution for the random equation of degree 5 with one unknown (50) was found. However, to solve the function (51) is beyond my ability, therefore, I leave this puzzle to the clever reader.

For example, Let's find the solution of function (52):

$$
\begin{aligned}
& t^{5}+2 t^{4}+3 t^{3}+4 t^{2}+5 t-6=0 \\
& a=\frac{A_{4}}{5 A_{5}}=\frac{2}{5} \\
& c=\frac{A_{2}-A_{5} \times 10 a^{3}}{3\left(A_{3}-A_{5} \times 10 a^{2}\right)}=\frac{4-10 \times \frac{8}{125}}{3\left(3-10 \times \frac{4}{25}\right)}=\frac{4}{5} \\
& p=\frac{A_{3}-A_{5} \times 10 a^{2}}{A_{5}}=\frac{7}{5} \\
& q=\frac{\left(A_{3}-A_{5} \times 10 a^{2}\right) \times 3(c-a)}{A_{5}}=\frac{42}{25} \\
& r=\frac{\left(A_{3}-A_{5} \times 10 a^{2}\right) \times 3(c-a)^{2}+\left[A_{1}-A_{5} \times 5 a^{4}-\left(A_{3}-A_{5} \times 10 a^{2}\right) \times 3 c^{2}\right]}{A_{5}}=\frac{357}{125} \\
& s=\frac{\left(A_{3}-A_{5} \times 10 a^{2}\right)(c-a)^{3}-a\left[A_{1}-A_{5} \times 5 a^{4}-\left(A_{3}-A_{5} \times 10 a^{2}\right) \times 3 c^{2}\right]-B-A_{5} a^{5}-\left(A_{3}-A_{5} \times 10 a^{2}\right) c^{3}}{A_{5}}=-7.49056 \\
& e-d^{2}+g=\frac{7}{5} \\
& f-d e+d g=\frac{42}{25}
\end{aligned}
$$




$$
\begin{gathered}
-d f+e g=\frac{357}{125} \\
f g=-7.49056
\end{gathered}
$$

If we can find the value of $d, e, f, g$, then the solution for the Equation (52) was found. Obviously, the value of $d, e, f, g$ can't be positive integer, then the solution $t$ of function (52) can't be positive integer.

Similarly, if $n=6$, the function (26) is equal to the function (54) shows below:

$$
\begin{gathered}
t^{5}+6 a t^{4}+15 a^{2} t^{3}+20 a^{3} t^{2}+15 a^{4} t^{1}-\left(m-6 a^{5}\right)=0 \\
a^{\prime}=\frac{A_{4}}{5 A_{5}}=\frac{6}{5} a \\
c^{\prime}=\frac{A_{2}-A_{5} \times 10 a^{\prime 3}}{3\left(A_{3}-A_{5} \times 10 a^{\prime 2}\right)}=\frac{20 a^{3}-10 \times \frac{216}{125} a^{3}}{3\left(15 a^{2}-10 \times \frac{36}{25} a^{2}\right)}=-\frac{4}{15} a \\
q=\frac{\left(A_{3}-A_{5} \times 10 a^{\prime 2}\right) \times 3\left(c^{\prime}-a^{\prime}\right)}{A_{5}}=-\frac{66}{25} a^{3} \\
S=\frac{\left(A_{3}-A_{5} \times 10 a^{\prime 2}\right)\left(c^{\prime}-a^{\prime}\right)^{3}-a^{\prime}\left[A_{1}-A_{5} \times 5 a^{\prime 2}-\left(A_{3}-A_{5} \times 10 a^{\prime 2}\right) \times 3 c^{\prime 2}\right]-B-A_{5} a^{\prime 5}-\left(A_{3}-A_{5} \times 10 a^{\prime 2}\right) c^{\prime 3}}{A_{5}} \\
\left.=-\left(9.77472 a^{5}+B\right)=-\left(3.77472 a^{5}+m\right) \quad A_{5} \times 10 a^{\prime 2}\right) \times 3\left(c^{\prime}-a^{\prime}\right)^{2}+\left[A_{1}-A_{5} \times 5 a^{\prime 4}-\left(A_{3}-A_{5} \times 10 a^{\prime 2}\right) \times 3 c^{\prime 2}\right] \\
A_{5}
\end{gathered}
$$

$a$ and $m$ are positive integer, if $p, q, r, s$ are positive integer, then the solution $t$ of function (54) can be a positive integer. Therefore, $a=5 k, k$ is positive integer, so:

$$
\begin{gathered}
p=\frac{3}{5} a^{2}=15 k^{2} \\
q=-\frac{66}{25} a^{3}=-330 k^{3} \\
r=\frac{1047}{125} a^{4}=5235 k^{4} \\
s=-\left(3.77472 a^{5}+m\right)=-\left(11796 k^{5}+m\right) \\
e-d^{2}+g=15 k^{2} \\
f-d e+d g=-330 k^{3} \\
-d f+e g=5235 k^{4} \\
f g=-\left(11796 k^{5}+m\right)
\end{gathered}
$$


If we can solve the function (51), then we can find the relationship between $d$, $e, f, g$ and $k$ through function (55), then we can according to the result in Part one to judge if the function (54) can have a positive integer solution.

However, the function (26) can be further transformed to the function (26-1)

$$
t^{n-2}+C_{n}^{(n-1)} a^{1} t^{(n-3)}+\cdots+C_{n}^{3} a^{(n-3)} t^{1}+C_{n}^{2} a^{(n-2)} t^{0}+C_{n}^{1} a^{(n-1)}-\frac{m}{t}=0
$$

Thus, the function (54) can be deformed to function (56):

$$
t^{4}+6 a t^{3}+15 a^{2} t^{2}+20 a^{3} t^{1}+15 a^{4}-\frac{m-6 a^{5}}{t}=0
$$

Let $\frac{m-6 a^{5}}{t}=m^{\prime}$, then we can use the result in Part two to prove the value of $t$ in function (56) cannot be a positive integer. What's more, the function (56) can be transformed further to function (57):

$$
t^{3}+6 a t^{2}+15 a^{2} t^{1}+20 a^{3}-\frac{m^{\prime}-15 a^{4}}{t}=0
$$

Let $\frac{m^{\prime}-15 a^{4}}{t}=m^{\prime \prime}$, then we can use the result in Part one to prove the value of $t$ in function (57) cannot be a positive integer.

$$
\begin{gathered}
t^{3}+6 a t^{2}+15 a^{2} t^{1}-\left(m^{\prime \prime}-20 a^{3}\right)=0 \\
p=\frac{3 A_{1} A_{3}-A_{2}^{2}}{3 A_{3}^{2}}=3 a^{2} \\
q=-\frac{27 A_{3}^{2} B+9 A_{1} A_{2} A_{3}-2 A_{2}^{3}}{27 A_{3}^{3}}=6 a^{3}-m^{\prime \prime} \\
w=\sqrt[3]{-\frac{q}{2}+\sqrt{\left(\frac{q}{2}\right)^{2}+\left(\frac{p}{3}\right)^{3}}}+\sqrt[3]{-\frac{q}{2}-\sqrt{\left(\frac{q}{2}\right)^{2}+\left(\frac{p}{3}\right)^{3}}} \\
u=\sqrt{\left(\frac{q}{2}\right)^{2}+\left(\frac{p}{3}\right)^{3}}=\sqrt{\left(3 a^{3}-\frac{m^{\prime \prime}}{2}\right)^{2}+\left(a^{2}\right)^{3}}
\end{gathered}
$$

The value of $u$ in the function (58) must be a integer, therefore, $\left(3 a^{3}-\frac{m^{\prime \prime}}{2}\right)^{2}+\left(a^{2}\right)^{3}$ must be a positive square integer, otherwise, the value of $w$ can't be a rational number.

Let $m^{\prime \prime}=2 k, k$ is positive integer, then:

$$
u=\sqrt{\left(3 a^{3}-\frac{m^{\prime \prime}}{2}\right)^{2}+\left(a^{2}\right)^{3}}=\sqrt{\left(3 a^{3}-k\right)^{2}+\left(a^{2}\right)^{3}}
$$

Obviously, if $\left(3 a^{3}-k\right)^{2}=K^{2} a^{6}, K^{2}+1=R^{2}, K$ and $R$ is a positive integer, under this condition, the value of $u$ can be a integer. Then, $K^{2}=0,3,8,15, \cdots$, $R=1,2,3,4, \cdots$, and $u= \pm R a^{3}$. However, the value of $K$ must be a positive square integer, a positive square integerplus 1 is still a square integer, only when $K^{2}=0, R^{2}=1$ can satisfy the requirement, then $k=3 a^{3}, m^{\prime \prime}=2 k=6 a^{3}$, 
$u= \pm a^{3}$.

$$
w=\sqrt[3]{0+\left( \pm a^{3}\right)}+\sqrt[3]{0-\left( \pm a^{3}\right)}=0
$$

Therefore, the solution of $t$ satisfy the function in (57) is $t=-\frac{A_{2}}{3 A_{3}}+w=-2 a+w=-2 a$. However, the

$$
\begin{aligned}
\frac{m^{\prime}-15 a^{4}}{t} & =m^{\prime \prime}=6 a^{3} \\
\frac{m-6 a^{5}}{t} & =m^{\prime} \\
\frac{b^{n}}{t} & =m
\end{aligned}
$$

According to the 3 equation infunction (59), we can get:

$$
6 a^{3} t^{3}+15 a^{4} t^{2}+6 a^{5} t^{1}-b^{n}=0
$$

Substitute $t=-2 a$ into the function (60), then $b^{n}=0$, thus $b=0$, this is contradictory with $b>0$, therefore, the value of $t$ in function (57) can't be positive integer, thus, the Fermat's Last Theorem is established when $n=6$.

Through this method, we can prove the Fermat's Last Theorem is established when $n=6$ even we don't know the solution of equation of degree 5 with one unknown, what's more, we can use the same method to prove the Fermat's Last Theorem is established when $n>6$, thus the Fermat's Last Theorem was proved.

Use the way we transform the function (26) to function (26-1), we can finally transform the function (26) to function (26-2):

$$
\begin{aligned}
& t^{3}+C_{n}^{(n-1)} a^{1} t^{2}+C_{n}^{(n-2)} a^{2} t^{1}-\left(m^{\prime \prime}-C_{n}^{(n-3)} a^{3}\right)=0 \\
& m^{\prime \prime}=\frac{m}{t^{n-4}}-\frac{C_{n}^{1} a^{(n-1)}}{t^{n-4}}-\frac{C_{n}^{2} a^{(n-2)}}{t^{n-5}}-\cdots-\frac{C_{n}^{(n-4)} a^{4}}{t^{1}}
\end{aligned}
$$

The function (26-2) is equal to:

$$
\begin{gathered}
t^{3}+n a^{1} t^{2}+\frac{n(n-1)}{2} a^{2} t^{1}-\left(m^{\prime \prime}-\frac{n(n-1)(n-2)}{6} a^{3}\right)=0 \\
p=\frac{3 A_{1} A_{3}-A_{2}^{2}}{3 A_{3}^{2}}=\frac{n(n-3)}{6} a^{2} \\
q=-\frac{27 A_{3}^{2} B+9 A_{1} A_{2} A_{3}-2 A_{2}^{3}}{27 A_{3}^{3}}=\frac{n(n-3)(2 n-3)}{27} a^{3}-m^{\prime \prime} \\
w=\sqrt[3]{-\frac{q}{2}+\sqrt{\left(\frac{q}{2}\right)^{2}+\left(\frac{p}{3}\right)^{3}}}+\sqrt[3]{-\frac{q}{2}-\sqrt{\left(\frac{q}{2}\right)^{2}+\left(\frac{p}{3}\right)^{3}}} \\
u=\sqrt{\left(\frac{q}{2}\right)^{2}+\left(\frac{p}{3}\right)^{3}}=\sqrt{\left(\frac{n(n-3)(2 n-3)}{54} a^{3}-\frac{m^{\prime \prime}}{2}\right)^{2}+\left(\frac{n(n-3)}{18} a^{2}\right)^{3}}
\end{gathered}
$$

The value of $u$ in the function (61) must be a integer, therefore, 
$\Delta=\left(\frac{n(n-3)(2 n-3)}{54} a^{3}-\frac{m^{\prime \prime}}{2}\right)^{2}+\left(\frac{n(n-3)}{18} a^{2}\right)^{3}$ must be a positive square integer, otherwise, the value of $w$ can't be a rational number.

Let $a=3 s, m^{\prime \prime}=2 k s^{3}, s$ and $k$ is positive integer, then:

$$
u=\sqrt{\left(\frac{n(n-3)(2 n-3)}{2} s^{3}-k s^{3}\right)^{2}+\left(\frac{n(n-3)}{2} s^{2}\right)^{3}}
$$

$\frac{n(n-3)(2 n-3)}{2}$ and $\frac{n(n-3)}{2}$ are integer when $n$ is integer and $n>3$.

Let $\frac{n(n-3)}{2}=r$, and $r$ is a positive integer $(r=2,5,9,14,20, \cdots)$, then $\Delta=\left((2 n-3) r s^{3}-k s^{3}\right)^{2}+\left(r s^{2}\right)^{3}$

Obviously, if $\left((2 n-3) r s^{3}-k s^{3}\right)^{2}=K^{2} s^{6}, K^{2}+r^{3}=R^{2}, K$ and $R$ is integer, under this condition, the value of $u$ can be a integer.

$$
\begin{gathered}
\frac{\Delta}{s^{6}}=((2 n-3) r-k)^{2}+r^{3}=R^{2}=K^{2}+r^{3} \\
r^{3}=R^{2}-K^{2}=(R+K)(R-K)
\end{gathered}
$$

Let $(R+K)=\frac{r^{2}}{m}$, then $(R-K)=m r$,

$R, k$ and $r$ are integer, then $m \in Q$

$$
\begin{aligned}
R & =\left(\frac{r^{2}}{m}+m r\right) / 2 \\
K & =\left(\frac{r^{2}}{m}-m r\right) / 2 \\
\frac{w}{s}=\sqrt[3]{-\frac{q}{2}+u}+\sqrt[3]{-\frac{q}{2}-u} & =\sqrt[3]{-K+R}+\sqrt[3]{-K-R}=\sqrt[3]{m r}+\sqrt[3]{-\frac{r^{2}}{m}}
\end{aligned}
$$

Only if $m=-\frac{N^{3}}{r}, N$ is integer but $N \neq 0, r=\frac{n(n-3)}{2}$, then $\frac{w}{s}=-N+\frac{r}{N}=-N+\frac{n(n-3)}{2 N}$ is rational, then $t=-\frac{n}{3} a+w=\left(-n-N+\frac{n(n-3)}{2 N}\right) s$ can be integer.

$$
\begin{aligned}
& R=\frac{\frac{r^{2}}{m}+m r}{2}=-\frac{\left(\frac{r}{N}\right)^{3}+N^{3}}{2}=-\frac{\left(\frac{n(n-3)}{2 N}\right)^{3}+N^{3}}{2} \\
& K=\frac{\frac{r^{2}}{m}-m r}{2}=-\frac{\left(\frac{r}{N}\right)^{3}-N^{3}}{2}=-\frac{\left(\frac{n(n-3)}{2 N}\right)^{3}-N^{3}}{2}
\end{aligned}
$$

The value of $N$ must be the factor of $r$, thus the value of $R, K$ could be integer. 
For example, if $n=7, r=\frac{n(n-3)}{2}=14$

$$
R=-\frac{\left(\frac{14}{N}\right)^{3}+N^{3}}{2}
$$

If $N=1,2,7,14,\left(\frac{14}{N}\right)^{3}+N^{3}$ is an odd number;

If $N$ is integer and $N \neq 0,1,2,7$, and $14,\left(\frac{14}{N}\right)^{3}+N^{3}$ is a fraction number, therefore, $R=-\frac{\left(\frac{14}{N}\right)^{3}+N^{3}}{2}$ can not be a integer, which is contradictory with $R$ is a integer. Thus $m \neq-\frac{N^{3}}{r}$ and $\frac{w}{s}$ is irrational, $s$ is integer, the value of $w$ is irrational, then the value of $t$ in function (26-2) can't be positive integer when $n=7$, so the Fermat's Last Theorem is established when $n=7$.

Similarly, if $\boldsymbol{n}=\mathbf{8}, \quad r=\frac{n(n-3)}{2}=20$

$$
R=-\frac{\left(\frac{20}{N}\right)^{3}+N^{3}}{2}
$$

If $N=1,2,4,5,10,20,\left(\frac{20}{N}\right)^{3}+N^{3}$ can be a integer, but only $N=2,10, R$ can be a integer.

When $N=2$ or $10, R=-504$, in other condition, $R$ is not a integer.

$$
\begin{gathered}
K=((2 n-3) r-k)=(260-k)=-\frac{\left(\frac{20}{N}\right)^{3}-N^{3}}{2}= \pm 496 \\
r^{3}=R^{2}-K^{2}=20^{3}=(-504)^{2}-( \pm 496)^{2} \\
\frac{w}{s}=-N+\frac{r}{N}= \pm 8 \\
t=-\frac{n}{3} a+w=\left(-n-N+\frac{n(n-3)}{2 N}\right) s
\end{gathered}
$$

When $N=2, \quad t=(-8-2+10) * s=0 * s=0$

When $N=10, t=(-8-10+2) * s=-16 * s<0$

which is contradictory with $t$ is a integer and $0<t<b$. so the Fermat's Last Theorem is established when $n=8$.

$$
\text { If } n=9, r=\frac{n(n-3)}{2}=27 \begin{aligned}
& \\
& \qquad R=-\frac{\left(\frac{27}{N}\right)^{3}+N^{3}}{2}
\end{aligned}
$$


If $N=1,3,9,27,\left(\frac{27}{N}\right)^{3}+N^{3}$ can be even number, and $R$ can be a integer.

$N=1, R=-9842, \quad K=-9841, \frac{w}{s}=26, \quad t=17 s$

$N=3, R=-378, \quad K=-351, \frac{w}{s}=6, \quad t=-3 s$

$N=9, R=-378, \quad K=351, \frac{w}{s}=6, \quad t=-15 s$

$N=27, R=-9842, \quad K=9841, \frac{w}{s}=-26, \quad t=-35 s$

Only if $N=1, t=17 \mathrm{~s}$ satisfies the condition. What's more, we have to verify if the value of $b$ is integer or not.

$$
\begin{gathered}
K=((2 n-3) r-k)=-9841, k=10246 \\
m^{\prime \prime}=2 k s^{3}=20592 s^{3} \\
m^{\prime \prime}=\frac{m}{t^{n-4}}-\frac{C_{n}^{1} a^{(n-1)}}{t^{n-4}}-\frac{C_{n}^{2} a^{(n-2)}}{t^{n-5}}-\cdots-\frac{C_{n}^{(n-4)} a^{4}}{t^{1}} \\
\frac{b^{n}}{t}=m, \quad a=3 s, \quad n=9, \quad t=17 s, \text { then: } \\
m^{\prime \prime}=\frac{b^{9}}{(17 s)^{6}}-\frac{9(3 s)^{8}}{(17 s)^{5}}-\frac{36(3 s)^{7}}{(17 s)^{4}}-\frac{84(3 s)^{6}}{(17 s)^{3}}-\frac{126(3 s)^{5}}{(17 s)^{2}}-\frac{126(3 s)^{4}}{(17 s)^{1}}=20592 s^{3} \\
b^{9}=9(3 s)^{8} *(17 s)+36(3 s)^{7} *(17 s)^{2}+84(3 s)^{6} *(17 s)^{3} \\
+126(3 s)^{5} *(17 s)^{4}+126(3 s)^{4} *(17 s)^{5}+20592 s^{3} *(17 s)^{6} \\
=514413737217 * s^{9} \\
b=\sqrt[9]{514413737217} s
\end{gathered}
$$

$s$ is a positive integer, thus $b$ is irrational, which is contradictory with $b$ is a integer. Therefore, the Fermat's Last Theorem is established when $n=9$.

If $n=10, r=\frac{n(n-3)}{2}=35$

Only if $N=1, t=24 s$ satisfies the condition.

Use the same way to verify if $b$ is a integer or not when $N=1, t=24 \mathrm{~s}$,

$$
\begin{gathered}
b^{10}=10(3 s)^{9}(24 s)^{1}+45(3 s)^{8} *(24 s)^{2}+120(3 s)^{7} *(24 s)^{3} \\
+210(3 s)^{6} *(24 s)^{4}+252(3 s)^{5} *(24 s)^{5} \\
+210(3 s)^{4} *(24 s)^{6}+44064 s^{3} *(24 s)^{7} \\
=205891132035600 * s^{10} \\
b=\sqrt[10]{205891132035600} s
\end{gathered}
$$

$s$ is a positive integer, thus $b$ is irrational, which is contradictory with $b$ is a integer. Therefore, the Fermat's Last Theorem is established when $n=10$.

The values of $b$ satisfy the condition under different $n$ is concluded in the Table 1 .

Table 1 indicated that if $2<n<21$, in order to have a positive integer solution 
Table 1. The value of $b$ under different $n$.

\begin{tabular}{|c|c|c|c|c|c|}
\hline$n$ & $r$ & $N$ & $k$ & $t$ & $b$ \\
\hline 3 & 0 & none & & & \\
\hline 4 & 2 & none & & & \\
\hline 5 & 5 & 1 & 97 & $-s$ & \\
\hline 6 & 9 & 1 & 445 & $2 s$ & $\sqrt[6]{14896} s$ \\
\hline 7 & 14 & none & & & \\
\hline 8 & 20 & $\begin{array}{l}2, \\
10\end{array}$ & $\begin{array}{l}756 \\
-236\end{array}$ & $\begin{array}{c}0 \\
-16 s\end{array}$ & \\
\hline 9 & 27 & 1 & 10,246 & $17 s$ & $\sqrt[9]{514413737217} s$ \\
\hline 10 & 35 & 1 & 22,032 & $24 s$ & $\sqrt[10]{205891132035600} s$ \\
\hline 11 & 44 & 2 & 6156 & $9 s$ & $\sqrt[11]{743008193541} s$ \\
\hline 12 & 54 & none & & & \\
\hline 13 & 65 & 1 & 138,807 & $51 s$ & $\sqrt[13]{33198531813531451984941} s$ \\
\hline 14 & 77 & 1 & 230,191 & $62 s$ & $\sqrt[14]{461540731532546208768008} s$ \\
\hline 15 & 90 & none & & & \\
\hline 16 & 104 & $\begin{array}{l}2 \\
4\end{array}$ & $\begin{array}{l}73,316 \\
11,772\end{array}$ & $\begin{array}{c}34 s \\
6 s\end{array}$ & $\begin{array}{c}\sqrt[16]{12337511914217166319227520} s \\
\sqrt[16]{1853020145805120 s} s\end{array}$ \\
\hline 17 & 119 & 1 & 846,268 & $101 s$ & $\sqrt[11]{19479004955562800041143429455772221} s$ \\
\hline 18 & 135 & $\begin{array}{l}1 \\
3 \\
5\end{array}$ & $\begin{array}{l}1,234,642 \\
50,004 \\
14,234\end{array}$ & $\begin{array}{c}116 s \\
24 s \\
4 s\end{array}$ & $\begin{array}{c}\sqrt[18]{23015822943866122205667120134071432} s \\
\sqrt[18]{58149737003040059302969680} s \\
\sqrt[18]{1628413210489960} s\end{array}$ \\
\hline 19 & 152 & $\begin{array}{l}2 \\
4\end{array}$ & $\begin{array}{c}224,804 \\
32,724\end{array}$ & $\begin{array}{l}55 s, \\
15 s\end{array}$ & $\begin{array}{c}\sqrt[11]{3199866632452173458088314772999205 s} \\
\sqrt[19]{708235345355336514096165 s}\end{array}$ \\
\hline 20 & 170 & none & & & \\
\hline
\end{tabular}

Note: "none" means there is no integer of $N$ to make $R=-\frac{\left(\frac{r}{N}\right)^{3}+N^{3}}{2}$ and $K=-\frac{\left(\frac{r}{N}\right)^{3}-N^{3}}{2}$ to be integer.

of $t$ in function (26-2), the value of $b$ cannot be a integer (Although the value of $b$ is very close to a integer), which means $b$ and $c(c=a+t)$ in function (1) can not be integer at the same time, thus the Fermat's Last Theorem is established when $2<n<21$.

Use the same way, we can easily prove the Fermat's Last Theorem is established under different indices $n$.

4) Part four: The conclusion of Section 2.3

In order to prove the value of $c$ satisfies the function (1) can't be positive integer when $a, b$, and $n$ are positive integer and $n>2$, in this section, we find the solution for the random equation of degree $n$ with one unknown (when $n=3$ and 4), and proved that the Fermat's theorem was established at $n=3,4$ and 5, 
the solution for the random equation of degree 5 with one unknown is hard to be found, if we find this solution, we can prove that the Fermat's theorem was established at $n=6$. Therefore, if we want to prove the Fermat's theorem, we have to find the solution for the random equation of degree $n$ with one unknown, then we can solve the certain function (26)

$$
t^{n-1}+C_{n}^{(n-1)} a^{1} t^{(n-2)}+\cdots+C_{n}^{2} a^{(n-2)} t^{1}+C_{n}^{1} a^{(n-1)}-m=0
$$

has no positive integer solution, thus the Fermat's theorem was proved.

However, if we can't find the solution for the random equation of degree $n$ with one unknown when $n>4$, we can transfer the function (26) into (26-2)

$$
\begin{gathered}
t^{3}+C_{n}^{(n-1)} a^{1} t^{2}+C_{n}^{(n-2)} a^{2} t^{1}-\left(m^{\prime \prime}-C_{n}^{(n-3)} a^{3}\right)=0 \\
m^{\prime \prime}=\frac{m}{t^{n-4}}-\frac{C_{n}^{1} a^{(n-1)}}{t^{n-4}}-\frac{C_{n}^{2} a^{(n-2)}}{t^{n-5}}-\cdots-\frac{C_{n}^{(n-4)} a^{4}}{t^{1}}
\end{gathered}
$$

thus we proved the Fermat's Last Theorem is established when $n=3,4,5, \ldots \ldots$, 20 in this section, and in this method, we can also prove the Fermat's Last Theorem is established when $n>20$, then the Fermat's theorem was proved.

\section{Extension of Fermat's Last Theorem}

Based on the Fermat's Last Theorem, I put forward another extension theorem:

$$
\begin{aligned}
& \sum_{i=1}^{i=k} a_{i}^{n}=a_{1}^{n}+a_{2}^{n}+\cdots+a_{k}^{n}=b^{n} \\
& \sum_{i=1}^{i=n} a_{i}^{n}=a_{1}^{n}+a_{2}^{n}+\cdots+a_{n}^{n}=b^{n}
\end{aligned}
$$

When $n$ is integer and $n>2$, to satisfy the function $(E-1)$ and $(E-2)$, $a_{1}, a_{2}, \cdots, a_{k} \quad(k=2,3, \cdots$, when $k=2$, function ( $E-1)$ is equivalent to the Fermat's Last Theorem)and $a_{1}, a_{2}, \cdots, a_{n} \quad(n=3,4, \cdots)$ and $b$ can't be positive integer at the same time.

The function $a_{1}^{3}+a_{2}^{3}+a_{3}^{3}=b^{3}$ has positive integer solution, so the key of the extension theorem is to find: under what conditions, the function $(E-1)$ and $(E-2)$ have no positive integer solution?

\section{Conclusions}

In this paper, I proposed an easy way to prove the Fermat's Last Theorem through a geometric method, and I found the relationship between $a^{n}+b^{n}=c^{n}=(a+t)^{n}$ and the solution $t$ for equation of degree $n$ with one unknown, then I found the solution when $n=3$ and 4 . If we can't find the solution of function (26) when $n>5$,

$$
\begin{gathered}
t^{n-1}+C_{n}^{(n-1)} a^{1} t^{(n-2)}+\cdots+C_{n}^{2} a^{(n-2)} t^{1}+C_{n}^{1} a^{(n-1)}-m=0 \\
m=\frac{b^{n}}{t}
\end{gathered}
$$

We can transfer function (26) to (26-2) 


$$
\begin{aligned}
& t^{3}+C_{n}^{(n-1)} a^{1} t^{2}+C_{n}^{(n-2)} a^{2} t^{1}-\left(m^{\prime \prime}-C_{n}^{(n-3)} a^{3}\right)=0 \\
& m^{\prime \prime}=\frac{m}{t^{n-4}}-\frac{C_{n}^{1} a^{(n-1)}}{t^{n-4}}-\frac{C_{n}^{2} a^{(n-2)}}{t^{n-5}}-\cdots-\frac{C_{n}^{(n-4)} a^{4}}{t^{1}}
\end{aligned}
$$

In this way, we can easily prove the Fermat's Last Theorem is established under different indices $n$, and the value of $b$ that make $c=a+t$ to be a integer under different $n$ is:

When $n=3, b^{3}=2 k s^{3}=0$

When $n>3$,

$$
\begin{aligned}
& b^{n}= B s^{n}=(\zeta s)^{n} \\
&= C_{n}^{(n-1)}(3 s)^{(n-1)}(t)^{1}+C_{n}^{(n-2)}(3 s)^{(n-2)}(t)^{2} \\
&+\cdots+C_{n}^{4}(3 s)^{4} *(t)^{(n-4)}+2 k s^{3} *(t)^{(n-3)} \\
& k= \frac{n(n-3)(2 n-3)}{2}+\frac{\left(\frac{n(n-3)}{2 N}\right)^{3}+N^{3}}{2} \\
& t=\left(-n-N+\frac{n(n-3)}{2 N}\right) s
\end{aligned}
$$

Nis the factor of $r=\frac{n(n-3)}{2}$ that can make kand $t$ to be a positive integer, $s$ is a positive integer. $\zeta=\sqrt[n]{B}$ is irrational that very closing to a integer, thus $b=\zeta s$ cannot be a positive integer.

Therefore, the function $a^{n}+b^{n}=c^{n}$ can be established only under the following conditions:

1) When $\theta=0, a+b=c, n=1$

2) When $\theta=\frac{\pi}{2}, a^{2}+b^{2}=c^{2}, n=2$

3) When $n>2, a, b, c$ are more than 0 and $a>b$ : $\theta \in\left(0, \frac{\pi}{2}\right)$, $a^{n}+b^{n}<c^{n}, \theta \in\left(\frac{\pi}{2}+\arcsin \frac{b}{2 a}, \pi\right), a^{n}+b^{n}>c^{n}$, under this condition, the function $a^{n}+b^{n}=c^{n}$ cannot be established. $\theta \in\left(\frac{\pi}{2}, \frac{\pi}{2}+\arcsin \frac{b}{2 a}\right)$, there is possible to make $a^{n}+b^{n}=c^{n}$, but the value of $a, b$ and $c$ can't be positive integer at the same time

\section{Acknowledgements}

I sincerely thank my family for trusting me and thank my friends for encouraging me. Especially, I thank the reader for reading this paper to the end and your questions are welcomed to be asked.

\section{Conflicts of Interest}

The author declares no conflicts of interest regarding the publication of this paper. 


\section{References}

[1] https://www.daviddarling.info/encyclopedia/F/Fermats last theorem.html

[2] Taylor, R. and Wiles, A. (1995) Ring Theoretic Properties of Certain Hecke Algebras. Annals of Mathematics, 141, 553-572. https://doi.org/10.2307/2118560 http://abel.math.harvard.edu/ rtaylor/

[3] Bratu, N.I. (2008) On the FERMAT'S Last Theorem. A New Proof for the Cases N-3 and N-5. Octogan Math. Mag., 16.

[4] https://www.math.net/article/fermats-last-theorem

[5] Wiles, A. (1995) Modular Elliptic-Curves and Fermat's Last Theorem. Ann. Math., 141, 443-551. https://doi.org/10.2307/2118559

[6] https://www.mathsisfun.com/pythagoras.html

[7] Euclid (2002) Euclid's Elements (Later Printing Edition). Green Lion Press, Santa $\mathrm{Fe}, \mathrm{NM}$.

[8] https://en.academic.ru/dic.nsf/enwiki/152736/ 